\title{
The physical internet as a new supply chain paradigm: a systematic literature review and a comprehensive framework
}

PI as a new supply chain paradigm

\author{
Horst Treiblmaier \\ Department of International Management, MODUL University Vienna, \\ Vienna, Austria \\ Kristijan Mirkovski
}

Department of Information Systems and Business Analytics, Deakin Business School, Burwood, Australia

Paul Benjamin Lowry

Pamplin College of Business, Virginia Tech, Blacksburg, Virginia, USA, and

Zach G. Zacharia

Department of Decision and Technology Analytics, Lehigh University, Bethlehem, Pennsylvania, USA

\begin{abstract}
Purpose - The physical internet (PI) is an emerging logistics and supply chain management (SCM) concept that draws on different technologies and areas of research, such as the Internet of Things (IoT) and key performance indicators, with the purpose of revolutionizing existing logistics and SCM practices. The growing literature on the PI and its noteworthy potential to be a disruptive innovation in the logistics industry call for a systematic literature review (SLR), which we conducted that defines the current state of the literature and outlines future research directions and approaches.

Design/methodology/approach - The SLR that was undertaken included journal publications, conference papers and proceedings, book excerpts, industry reports and white papers. We conducted descriptive, citation, thematic and methodological analyses to understand the evolution of PI literature.

Findings - Based on the literature review and analyses, we proposed a comprehensive framework that structures the PI domain and outlines future directions for logistics and SCM researchers.

Research limitations/implications - Our research findings are limited by the relatively low number of journal publications, as the PI is a new field of inquiry that is composed primarily of conference papers and proceedings.

Originality/value - The proposed PI-based framework identifies seven PI themes, including the respective facilitators and barriers, which can inform researchers and practitioners on future potentially disruptive SC strategies.
\end{abstract}

Keywords Physical Internet (PI), Systematic literature review (SLR), Citation analysis, Thematic analysis, Methodological analysis, Logistics, Supply chain management (SCM)

Paper type Literature review

(C) Horst Treiblmaier, Kristijan Mirkovski, Paul Benjamin Lowry and Zach G. Zacharia. Published by Emerald Publishing Limited. This article is published under the Creative Commons Attribution (CC BY 4.0) licence. Anyone may reproduce, distribute, translate and create derivative works of this article (for both commercial and non-commercial purposes), subject to full attribution to the original publication and authors. The full terms of this licence may be seen at http://creativecommons.org/licences/by/4.0/ legalcode.

Horst Treiblmaier and Kristijan Mirkovski share equal contribution for this study.

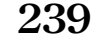

Received 14 November 2018

Revised 15 April 2019 1 July 2019

17 October 2019

11 December 2019 Accepted 11 December 2019 
IJLM
31,2

240

\section{Introduction}

The current global logistics and supply chain management (SCM) practices of transporting, storing and handling physical freight are unsustainable in the long run from economic, environmental and societal perspectives. Montreuil (2011, p. 71) identified 13 specific symptoms of the unsustainable global logistics and SCM practices that constitute the "worldwide global logistics sustainability grand challenge." However, using the latest improvements in information sharing, interconnectivity, information technology, data mining, big data and data analytics, it is possible to develop new logistics and SCM practices that are sustainable in the long run. The Physical Internet (PI) is a new boundary-spanning area of research and practice that seeks to address this grand challenge. The aim of the PI is to optimize logistics and SCM processes so as to create more efficient, effective and sustainable supply chains (SCs), which may lead to innovation of SC models and strategies (Kache and Seuring, 2017).

The PI is described as "a global logistics system based on the interconnection of logistics networks by a standardized set of collaboration protocols, modular containers, and smart interfaces for increased efficiency and sustainability" (Ballot et al., 2014, p. 23). Treiblmaier (2019, p. 3) defined the PI as "a comprehensive and measurable supply chain framework which is based on a network of physical components. These components are standardized as well as optimized and exchange information to improve the effectiveness, efficiency, and sustainability of supply chain management operations." The PI is a holistic concept that merges numerous relevant areas of logistics and SCM research with the promise of disrupting current logistics and SCM practices. These areas include sustainability, effectiveness and efficiency of global value chains and information flows, as well as horizontal and vertical collaboration. For example, the PI modular containers are "world-standard, smart, ecofriendly, and modular" aiming to transform the existing transport, handling and storage of cargo containers through "smart, sustainable, and seamless automation and human handling" (Montreuil et al., 2013a, p. 156). Similarly, PI hubs enable efficient unimodal and multimodal dispatching and routing of modular containers in an open logistics network via consolidation of loads, which in turn lowers inventory and logistics systems costs (Venkatadri et al., 2016).

Considering the growing number of PI publications and the globally increasing investments in PI-related projects, the PI is rapidly gaining relevance in both academic and practitioner circles. For example, the European Union has launched (1) the Modular Logistics Units in Shared Co-Modal Networks (MODULUSHCA) project (Huschebeck, 2012) and (2) the Alliance for Logistics Innovation through Collaboration in Europe (ALICE) platform (ALICE, 2014a). ALICE has set the following PI-related goals: (1) establish the landscape by elaborating, developing and demonstrating a PI-enabled interconnected logistics vision and its characteristics; (2) provide simulation- and field-based proof of concept by gradually implementing and testing key functions of PI-enabled interconnected logistics; and (3) develop a comprehensive strategy for research, innovation, and market deployment of logistics and SCM innovation in Europe (ALICE, 2014a).

The PI draws on concepts from numerous different technologies and areas of research, including the Internet of Things (IoT) for interconnectedness of logistics networks and the key performance indicators for continuous measurement and improvement. The PI has the potential to become a disruptive innovation that inspires vast multidisciplinary collaboration to solve several pressing social and business problems by revolutionizing extant logistics and SCM practices (Kache and Seuring, 2017). Christensen (2013) defined a disruptive innovation as a product or service that initially underperforms relative to the established products or services but eventually replaces them. According to Danneels (2004), disruptive innovation changes the basis of competition by changing the metrics along which organizations compete. Following the same line of reasoning, the PI is an emerging concept that currently 
underperforms in comparison to existing logistics and SCM practices but has the potential to radically transform the way physical products are being handled, transported and stored. These changes could greatly influence the environment, economy and society.

The PI is still a relatively new idea that is poorly understood. The current PI literature is largely unstructured and scattered across various academic disciplines. We suggest three reasons for the largely disjointed nature of the PI literature. First, the PI is a novel concept with articles published in various disciplines, including logistics, SCM and operations/ production management. Second, a substantial number of publications, including white papers, project deliverables and reports, have been published in industry-focused outlets. Third, numerous PI publications are "spin-offs" from industry projects and thus lack theoretical contributions. More importantly, given the emerging nature of the PI concept, there is a limited number of literature reviews. Using the technology adoption of firms as a theoretical framework, Sternberg and Norrman (2017) conducted a critical literature review of the PI in which they showed how (1) the perceived benefits in term of the novel PI business models, (2) the organizational readiness in terms of the PI technological blueprints, and (3) the external pressure in terms of the expected PI effects contribute to the adoption of the PI. Treiblmaier et al. (2016) provided a brief overview of the current PI literature in which they highlighted important opportunities in four main research areas: (1) the implementation of the PI in Europe and the United States, (2) the optimization of operations in PI hubs, (3) centralized reputation-based transportation auction, and (4) the impacts and challenges related to the shifts to the open logistics networks.

However, the existing literature review attempts are incomplete for two reasons. First, many PI publications in journals, books, conferences and reports have been omitted, which are important for providing a comprehensive analysis of the PI literature. Second, the evolution of the PI literature, including the under-researched themes and under-utilized methods, has not been addressed, which is necessary for laying down the directions of future PI research. Thus, a need exists for a holistic and inclusive review of the PI literature. Without a systematic literature review (SLR) of the existing PI literature to identify future research areas and methods, the PI research is unlikely to grow, possibly reducing research opportunities related to the exploration of new, innovative SC models and strategies. In line with Kache and Seuring's (2017) call for research on emerging concepts in SCM, our SLR makes three major contributions to the logistics and SCM research: (1) identification of major PI literature themes and methods, (2) creation of a comprehensive PI-themed framework, and (3) suggestions for future research directions and approaches regarding the PI. We also provide supplementary figures and tables in Appendix 1 and a comprehensive summary of the reviewed PI literature in Appendix 2.

In the following section, we describe the PI and its relevance to the logistics and SCM research. Next, we outline our review methodology. In the results section, we then provide descriptive, citation, thematic and methodological analyses. We then present our PI-themed framework, outline the future research directions and approaches and discuss contributions to the PI literature, as well as managerial implications. We conclude with the limitations of our literature review.

\section{The Physical Internet}

The term "Physical Internet" (PI) was first used in a 2006 headline of the British popular press magazine The Economist, which contained a survey of logistics and a variety of mainstream SC articles (Markillie, 2006). In the subsequent years, this publication inspired a team of researchers to explore the possibility of organizing the flow of physical goods in a manner similar to the organization of data flow on the digital Internet (Montreuil, 2011), leading to the emergence of the PI.
PI as a new supply chain paradigm 
IJLM
31,2

\section{The PI foundations}

The PI was conceptualized as a response to the inefficiencies and unsustainability of current logistics and SCM practices. Montreuil (2011, p. 74) described 13 issues that make the current logistics practices unsustainable: (1) limited space utilization for road, rail, sea and air transport; (2) empty travel being the norm rather than the exception; (3) poor working conditions for truck drivers; (4) products sitting idle; (5) inefficiency of product distribution; (6) inefficient use of production and storage facilities; (7) mediocre coordination within distribution networks; (8) high inefficiency of multimodal transportation; (9) dysfunctional city logistics; (10) inefficient cross-docking operations; (11) low network security and robustness; (12) difficulty justifying the use of IT in SCs; and (13) limited innovation opportunities. In light of these issues, Montreuil (2010) proposed 13 PI principles to address the "global logistics sustainability grand challenge": instrumentality, responsibility, metasystematization, openness, universality, interconnectivity, uniformity, accessibility, uniqueness, encapsulation, agentification, contracting and certification. He illustrated how to apply each of these principles, respectively, to achieve more sustainable supply chains. Accordingly, the PI is "an open global logistics system founded on physical, digital and operational interconnectivity through encapsulation, interfaces, and protocols [and] a perpetually evolving system driven by technological, infrastructural, and business innovation” (Montreuil et al., 2013a, p. 151).

\section{The PI objectives and constituents}

The main PI objective is to transform "the way physical objects are handled, moved, stored, realized, supplied, and used, aiming towards global logistics efficiency and sustainability" (Hakimi et al., 2012, p. 1). The PI aims to organize the transportation of physical goods in a manner similar to the way in which data packages are moved on the digital Internet. By sharing resources, such as vehicles and data, and designing transit centers, which enable seamless interoperability, the transportation of goods will be optimized with regard to cost, speed, efficiency and sustainability. To achieve this optimization, the PI sets common and universally agreed-upon standards and protocols to facilitate horizontal and vertical cooperation between organizations.

It is crucial to distinguish clearly between the PI and its constituents. The PI can be seen as an application of the IoT (Gubbi et al., 2013) that describes a network of physical objects embedded with technology to collect and exchange data. Similarly, the PI drives the creation of innovative delivery channels for services, whether in the area of data exchange or logistics, both of which are commonly subsumed under the term Internet of Services (Schroth and Janner, 2007). Additionally, the PI is an application of cloud computing (Armbrust et al., 2010) that is the practice of using a network of remote Internet servers, rather than a local server or personal computer, to store, manage and process data. Accordingly, the PI is an open concept that embraces novel technologies with the aim of disrupting the current logistics and SCM practices to design more efficient and effective SCs.

\section{The PI functionality and applications}

As noted, the PI does not directly manipulate physical goods but rather manipulates and manages the shipping containers that store goods, just like digital Internet packets store embedded data (Montreuil, 2011). For the PI to become full-fledged, numerous elements need to be coordinated, including physical objects, such as PI modular containers or PI transit centers, and more abstract concepts, such as legislation and business models. However, previous studies, which focused running simulations with a small number of actors in dedicated industries, have shown that considerable gains can be achieved through the application of the PI, even if it is incomplete. For example, Sarraj et al. (2014a) who used data representing the flow of goods from the fast-moving consumer goods (FMCG) industry in 
France to test various transportation protocols and scenarios, concluded that the "PI is very efficient within the FMCG supply networks of two large retailers" (p. 3206) and reported positive effects on greenhouse gas emissions, cost, lead time and travel delivery time. Similarly, Ballot et al. (2014) reported the findings of an exploration project in Canada in which simulations revealed various positive effects of the PI, including increased fill rates, energy savings, decreased transportation costs and reductions in the total logistics costs.

\section{Methodology}

The purpose of this SLR is to organize and categorize the state-of-the-art PI literature published in peer-reviewed journals, books, conference papers and proceedings, industry reports and white papers. Generally, the purpose of literature reviews is to map, consolidate and evaluate the intellectual territory of a certain field and to identify gaps to be filled with a view to further develop the existing body of knowledge (Tranfield et al., 2003). The current state of the PI literature can be characterized as dispersed because the literature appears in outlets from several different disciplines (e.g. logistics, SCM and operations/production management) and in outlets with a strong focus on practice rather than theory; thus, an SRL of the PI's current state is highly valuable. Such a review can provide better conceptualizations through an improved, common nomenclature and help identify compelling and clear directions for new applications in practice and for future PI research. In turn, this can inspire and support research efforts to improve existing SC models and strategies.

For this SLR, we followed the guidelines provided by Tranfield et al. (2003), Rousseau et al. (2008), Watson (2015), and Durach et al. (2017) to: (1) identify existing publications, (2) select and evaluate their contributions, (3) analyze and synthesize the data, (4) report on the findings and (5) propose a research agenda. We followed a systematic review process by (1) searching publications for potential inclusion; (2) selecting publications for inclusion or exclusion; (3) synthesizing selected publications in textual, tabular, or graphical form; and (4) critically analyzing contributions in terms of PI themes and methods (Watson, 2015). Next, we explain the search-and-selection and analytical processes used in this SLR.

\section{Search and selection process}

We produced a systematic review protocol that provides an overview of the four stages of the search-and-selection process (see Figure A1 in Appendix 1). In Stage 0, we undertook a preliminary informal search of a dedicated PI conference (i.e. the International PI Conference [IPIC]) from 2014 to 2018, aiming to identify whether a sufficient number of publications existed to enable us to conduct a SLR). This conference was a pertinent starting point because it is an open forum for academics and practitioners to explore, discuss and introduce cuttingedge concepts, methodologies, recent projects, technological advancements and start-up initiatives for current and future PI implementation (IPIC, 2018). We identified 62 publications - a sufficient number for the study - and our preliminary informal research informed the initial search process used in the next stage.

In Stage 1, we used several academic databases and search engines, such as ProQuest ${ }^{\mathrm{TM}}$, EBSCO $^{\mathrm{TM}}$, Emerald ${ }^{\mathrm{TM}}$ and Google Scholar ${ }^{\mathrm{TM}}$, to conduct the initial search. We also considered dedicated PI interest groups (e.g. the PI Initiative, ALICE and MODULUSHCA) and other relevant conferences (e.g. the International Conference on Industrial Engineering and Systems Management and the IFAC Symposium on Information Control in Manufacturing). We searched for the term "Physical Internet" in the titles, abstracts and introductions of the publications. Given the specificity of the subject, this was the only term that was needed to conduct a thorough search. The search process related to publications was limited to 20062019. We selected 2006 as the base year because the first article that ever mentioned the term PI was published in this year.

PI as a new supply chain paradigm 
IJLM 31,2

The main objective of the initial selection process was to include relevant publications based on a review of their titles, abstracts and introductions. We included publications dealing specifically with the PI concept and excluded those dealing with the physical infrastructure or computer hardware of the digital Internet. To further support the initial search process, we checked the references of the selected publications to identify additional publications for inclusion. We then combined the results of the two searches and removed duplicates. Whenever there was disagreement among the authors regarding the inclusion/ exclusion assessment, the publication in question was read and discussed until agreement was reached. Alternatively, publications were included provisionally and then assessed later in the selection process.

Stage 2 comprised the selection process, which entailed reading the included publications and making the final inclusion/exclusion decisions while applying a set of inclusion and exclusion criteria and extracting data for analysis. First, we read all the included publications in detail and carefully applied the inclusion and exclusion criteria. When dealing with a publication whose relevance was questionable, we notified each other and explained why it was unsuitable for inclusion. We either agreed to exclude the publication or engaged in further discussion until an agreement was reached. The inclusion criteria used in the selection process were the following:

(1) Publications whose main objective was to investigate the PI concept by conducting conceptual research (i.e. defining and refining the fundamental PI concepts), assessment research (i.e. assessing the PI's economic, environmental and societal impacts and sustainability improvements), solution design research (i.e. designing, engineering and testing methodologies and technologies for PI implementation), validation research (i.e. validating the implementation of the PI in real-world projects) or literature research (i.e. reviewed the existing PI literature). This inclusion criterion defines the primary scope of our study.

(2) Publications that had logistics or SCM as the core context. We included publications that address logistics and SCM issues related to the PI. This inclusion criterion was justified because the main contributions of this study are to the logistics and SCM literature.

(3) Publications that were written in English. The majority of the publications on the PI are written in English; however, a few have been written in French. This inclusion criterion was justified because most of the publications on the PI are written in English and we did not have French expertise on our team.

The exclusion criteria for the selection process were the following:

(1) Publications that discussed the unsustainable global logistics and SCs challenges (e.g. excessive carbon dioxide, resource wastage, inefficient business models) that led to the emergence of the PI. These publications were considered peripheral to the PI concept and were, therefore, excluded.

(2) Publications that addressed problems related to the interconnected logistics networks without making direct reference to the PI or drawing from its foundations and concepts. These publications were not directly linked to the PI and were, therefore, excluded.

(3) Publications for which only extended abstracts or PowerPoint presentations were available. These publications would not provide a sufficiently high level of detail for analysis in this study. 
(4) Publications that were found in the popular media that are descriptive in nature and whose main objective is to inform the public about the PI concept and its implementation. We excluded these publications because of their insignificant contributions to the PI literature and their lack of sufficient details for analysis.

Second, we extracted data from the included publications and organized the information in a tabular format (see Appendix 2). We extracted and organized data on each publication's author(s) and year, outlet, research methodology, research objective, research findings and conclusion and successive journal publication (for conference papers and proceedings only). Data extraction was conducted by two of the authors and then checked for completeness by the other two. Whenever there was disagreement regarding the correct classification of the papers, this was discussed among the authors until consensus was reached.

In Stage 3, we conducted a search validation to ensure that all the relevant publications were included in the systematic review. First, we double-checked the references of both the included and excluded publications and added any that had been missed. Second, we searched academic databases, search engines and dedicated interest groups and conferences again for the term "Physical Internet" and added any new publications. After completing the validation, we ended the search-and-selection process. The final dataset resulted in $192 \mathrm{PI}$ publications - 54 journal articles, 14 book sections, 97 conference proceedings and 27 reports and white papers - as outlined in Figure 1.

\section{Analysis process}

During the analysis process, we conducted descriptive, citation, thematic and methodological analyses of the 192 selected PI publications. First, we conducted a descriptive analysis to develop an overall understanding of the current state of the PI literature. We found that many PI publications employed multiple research methodologies (e.g. quantitative modeling combined with a simulation experiment) and therefore were assigned to a category and subcategory, depending solely on the contribution of each methodology. For example, a publication was categorized as "conceptual with simulation modeling" when its conceptualizations constituted a major contribution relative to the mathematical measurements and calculations within it. Moreover, when a simulation modeling was perceived as a "means to an end" to develop new or improved conceptualizations, then the
PI as a new supply chain paradigm

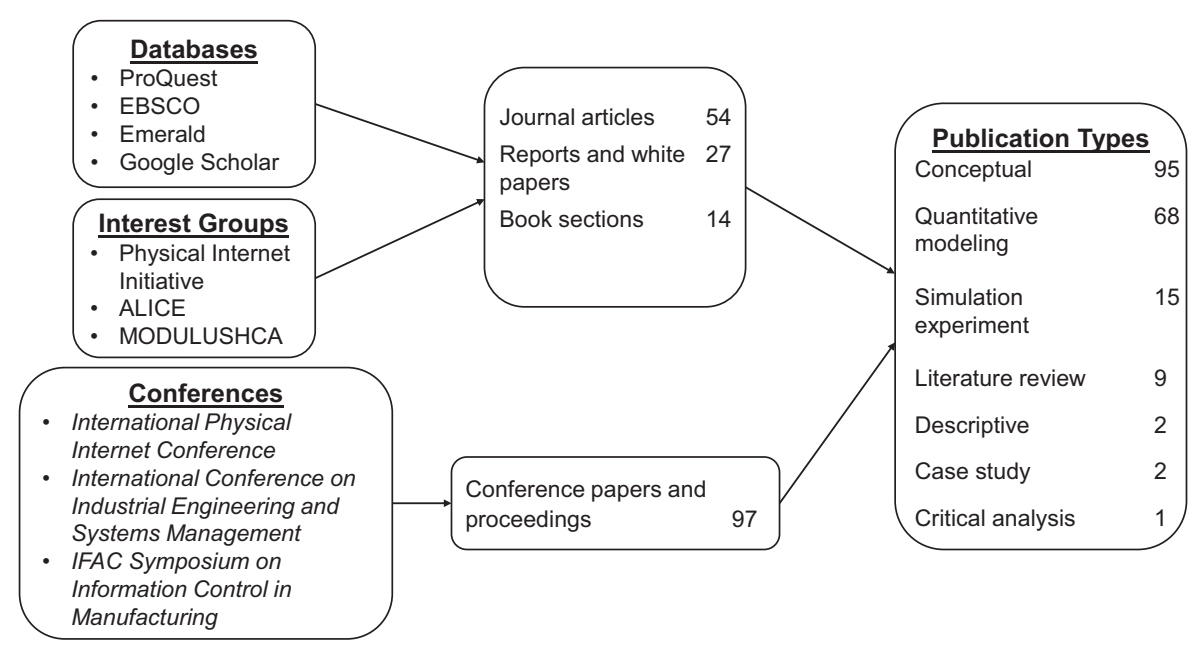

Figure 1.

PI literature review process 
IJLM

31,2

246

researchers determined that "conceptual development" was the major contribution of the publication. The categorization process was conducted independently by two researchers, who discussed any disagreements until full consensus was achieved, ensuring 100 percent interrater reliability.

Second, we conducted citation analysis using data from two databases: Scopus ${ }^{\mathrm{TM}}$ and Web of Science ${ }^{\mathrm{TM}}$. Scopus with 27 million citations and Web of Science with 36.1 million citations have long been identified as the two most extensive databases for citation analysis. Citation occurs when one publication refers to another as a source publication (Garfield, 1979). Out of the 192 selected publications, 31 publications with citations underwent citation analysis to identify influential publications and the inherent relationships existing among these publications. To achieve these objectives, we employed citation count and out-degree centrality measures. Degree centrality is measured by the number of direct links that a node has in a citation network. The more links it has, the more active or central it is. Nodes that are peripheral to the citation network are disadvantaged because they have fewer links relative to the central nodes. Out-degree centrality measures the number of publications that the focal publication cites (Freeman, 1978). After generating the citation network using the software package VOSviewer, we excluded the nodes without links, leaving us with a citation network of 22 publications from the period of 2014 to 2017.

Third, we classified each publication according to themes (Wilding et al., 2012; Beliën and Forcé, 2012; Olhager et al., 2015). First, we grouped the selected publications into seven PI themes: business models; cooperation models; legal models; seamless, secure and confidential data exchange; transit centers (hubs); vehicle usage optimization; and modular PI containers. We assigned one or more themes to each publication, depending on the coverage of each theme. For example, when a publication focused largely on solutions for PI transit centers and discussed modular containers as a part of the solution, the publication was assigned two themes: PI transit centers and modular containers. As researchers we independently assigned themes to publications, and whenever disagreements arose, we discussed the publication in question and arrived at a final decision. Using an iterative process, we synthesized the results by categorizing all PI publications into seven key themes as depicted in Figure 2.

Fourth, for our methodological analysis, we used a modified version of the PI classification scheme proposed by Pan et al. (2017). We selected this classification scheme because it provided a holistic perspective, which coincided with our objective to examine the evolution of the PI literature. We modified this classification scheme to include a "literature research" category to enable a more accurate categorization of the PI literature. Thus, we organized the selected publications into five categories: conceptual research, assessment research, solution design research, validation research and literature research. The categorization process was first conducted independently and then we discussed any areas of disagreement until a final decision was reached. Each publication was assigned to at least one method category. To make the categorization parsimonious and useful, we categorized some of the multiplemethod publications as single-method, depending on the significance of their contribution. For example, some assessment and solution design publications were categorized as solely solution design publications because their contributions were more significant than those related to their assessments. That is, when assessments were perceived as a "means to an end" for new or improved solution designs, then we determined that "solution designs" had a more significant contribution relative to "assessment."

Fifth, we further analyzed the content of the publications to understand the depth of knowledge contained in the PI literature. Based on this analysis, together with the identified themes and methods, we described the three stages of the PI literature evolution: incubation, exploration and expansion. More specifically, we independently analyzed the selected PI publications to determine their depth and complexity of analysis in relation to a theme or set of themes. Then, we categorized publications based on their similarity in terms of the depth 


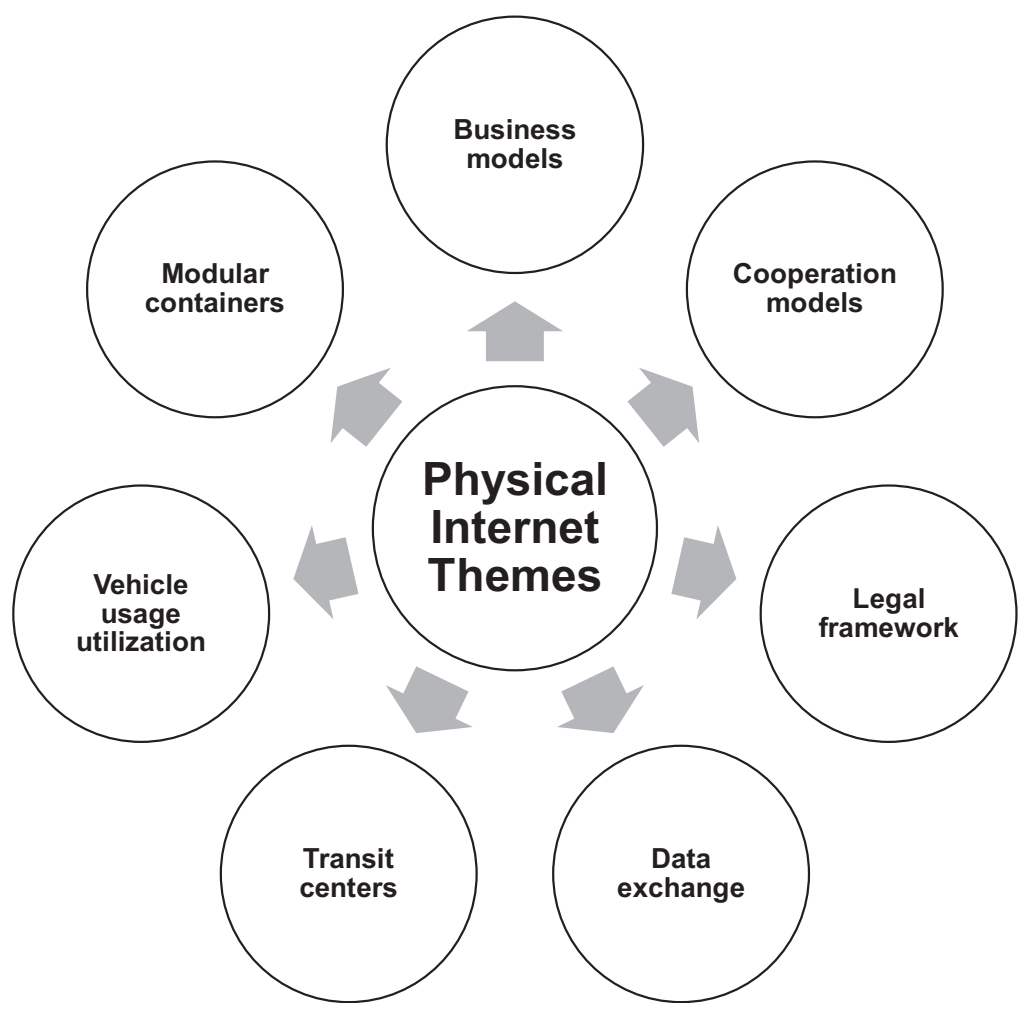

PI as a new supply chain paradigm

Figure 2. PI literature themes

and complexity of analysis. For example, purely conceptual publications, largely dealing with envisioning the PI concept, were grouped together. Similarly, publications that established the foundations of PI concepts, such as PI transit centers and modular containers, were classified into the same group. When there was a disagreement about how to classify a particular publication, we discussed the classification further until we came to a consensus. After the grouping of publications, we distilled the key characteristics of each group and name each according to a phase related to the evolution of the PI literature. Furthermore, we summarized the key elements - themes, facilitators and barriers - of the PI discussed in the literature and created a PI-based framework that guides future research. This process included techniques such as content and context analysis (Mayring, 2014; Glaser and Strauss, 1967).

\section{Results}

We now discuss the results of our literature review. We first discuss the descriptive analysis, which provides an overview of the PI literature. In the second section, we discuss the thematic analysis, which provides an overview of the main themes in the PI literature. In the third section, we discuss the methodological analysis, providing an overview of the main research methods used in the PI literature.

\section{Descriptive analysis}

The PI literature is continually growing. In total, we reviewed 148 articles, including academic, conference and commercial publications. The first publication on the PI dates back 
IJLM

31,2

\section{8}

to 2008 (see Figure A2 in Appendix 1). The number of conference and journal publications on the PI has been steadily increasing over the years - 2014 (31), 2015 (29) and 2016 (37) especially since the launch of a dedicated PI conference (i.e. IPIC). The rising number of PI publications over the years can also be attributed to the increased interest in this new concept in the corresponding academic and practitioner circles. However, this upward trend was interrupted in 2017, 2018 and 2019, from which there were 23, 21 and 23 publications, respectively. This partially suggests low conference-to-paper conversion rates as the research area matures.

Table I summarizes the types of PI publications. To date, the majority $(51 \%)$ have been conference papers and proceedings, with the number of journal articles (28\%) and book sections $(7 \%)$ being comparatively lower; however, these figures will likely increase, given the high number (89) conference publications from 2014 to 2019. More peer-reviewed journal publications on the PI have been published from 2016 to 2019 (51) than in all previous years (i.e. 2008-2015) combined (17). We also observed that only $14(7 \%)$ of the conference publications were later developed into journal publications. The remaining proportion of PI publications $(14 \%)$ take the form of reports for project deliverables and white papers targeting practitioner and government audiences.

Table II shows the research methodologies used in the reviewed PI publications. A large percentage of the publications are conceptual (49.5\%). Similarly, a large number of studies use quantitative modeling $(35 \%)$, including simulation experiments, computational experiments, Monte Carlo simulations and multi-agent-based simulations. A smaller percentage of the publications are descriptive (1\%), literature reviews $(5 \%)$ and simulation experiments without quantitative modeling $(8 \%)$. Furthermore, the PI literature currently has a relatively small number of publications that use case study (2) and critical analysis (1) as the sole research methodology.

Table III summarizes the academic outlets of PI publications. The International Journal of Production Research (17) has been the main outlet for PI journal publications, while the International PI Conference (72) has been the main outlet for PI conference publications. Moreover, Service Orientation in Holonic and Multi-Agent Manufacturing is the most significant peer-reviewed book for PI publications. Other peer-reviewed journals and conferences, such as Logistics Research (2), IEEE Access (2) Journal of Intelligent Manufacturing (2), the International Conference on Industrial Engineering and Systems Management (3) and the IFAC Symposium on Information Control Problems in Manufacturing (3), have published only a small number of PI publications.

\section{Citation analysis}

Of the 31 publications cited, 8 have at least 10 citations and 24 have fewer than 10 (see Table AI in Appendix 1). The most highly cited publications are Sarraj et al. (2014a), Sarraj

\begin{tabular}{lcc}
\hline Publication types & No. of publications & Percentage \\
\hline Commercial Publications & 27 & 14 \\
Reports & 25 & 13 \\
White papers & 2 & 1 \\
Conference Publications & 97 & 51 \\
Conference papers and proceedings & 97 & 51 \\
Academic Publications & 68 & 35 \\
Journal articles & 54 & 28 \\
Book sections & 14 & 7 \\
Total & 192 & 100 \\
\hline
\end{tabular}

Table I. Types of PI publications 
Research methodologies

Quantitative Modeling

Simulation experiment

Computational experiment

Case study

Monte Carlo simulation

Multi-agent based simulation

Regression analysis

Simple continuous-based model

Color Petri Nets

Querying analysis and regression model

Only quantitative modeling

Conceptual

Only conceptual

Simulation experiment

Case study

Functional design

Survey

Proof-of concept

Conceptual design

Structure analysis

Content analysis

Literature review

Simulation experiment

Only simulation experiment

Case study

Literature review

Only literature review

Case study

Survey

Field investigation and focus group

Descriptive

Only descriptive

Case study

Case study

Critical analysis

Total
No. of publications (\%)

$68(35 \%)$

36

19

3

3

2

249

$95(49.5 \%)$

48

15

16

3

3

2

2

1

1

$15(8 \%)$

12

3

$9(5 \%)$

6

1

1

$2(1 \%)$

1

$2(1 \%)$

$1(0.5 \%)$

$192(100 \%)$
PI as a new

supply chain

paradigm
Table II.

Research

methodologies of PI publications

\begin{tabular}{llcr}
\hline Academic outlets & & No. of publications & Year(s) of publication \\
\hline $\begin{array}{l}\text { Peer-reviewed } \\
\text { journals }\end{array}$ & $\begin{array}{l}\text { International Journal of Production } \\
\text { Research } \\
\text { Logistics Research }\end{array}$ & 17 & $2014-2017,2019$ \\
& $\begin{array}{l}\text { IEEE Access } \\
\text { Journal of Intelligent Manufacturing }\end{array}$ & 2 & 2011,2015 \\
Peer-reviewed & $\begin{array}{l}\text { Service Orientation in Holonic and Multi- } \\
\text { books }\end{array}$ & $\begin{array}{l}\text { Agent Manufacturing } \\
\text { Progress in Material Handling Research }\end{array}$ & $2018-2019$ \\
Peer-reviewed & $\begin{array}{l}\text { International Physical Internet } \\
\text { conferences }\end{array}$ & $\begin{array}{l}\text { Conference } \\
\text { International Conference on Industrial }\end{array}$ & 2014,2016 \\
& $\begin{array}{l}\text { Engineering and Systems Management } \\
\text { IFAC Symposium on Information }\end{array}$ & 32 & $2015-2016,2018-2019$ \\
& Control Problem in Manufacturing & 3 & $2014-2019$ \\
& & 2011,2013 & Academic outlets with \\
more than one PI \\
publication
\end{tabular}


IJLM

31,2

et al. (2014b), Montreuil et al. (2013a), with 25, 21 and 19 citations, respectively, indicating that they are major PI knowledge sources. Noticeably, in the past five years, the total number of citations has increased significantly from 42 in 2016 to 104 in 2017 and then to 60 halfway through 2018, suggesting that the PI literature is starting to establish a research community with sufficient critical mass in terms of researchers and practitioners willing to contribute to this phenomenon. The publications with the highest out-degree were Sternberg and Norrman (2017) and Sarraj et al. (2014a), with 14 and 10 links, respectively. Thus, by expanding the knowledge base to other publications, these two publications are vital to the dissemination of PI knowledge (see Figure A3 in Appendix 1).

With 93 citations, the International Journal of Production Research contains some of the most-cited publications, suggesting that this is the most important academic outlet for PI knowledge to date. We observed that seven $(26 \%)$ of the cited publications were nonempirical (i.e. conceptual, literature review and case study), suggesting that scholars are still attempting to make sense of the PI by elaborating on the basic concepts related to this new initiative. Conversely, there were $24(77 \%)$ empirical publications with citations (i.e. simulation experiments, computational experiments and Monte Carlo simulation), suggesting that researchers are making efforts to quantitatively investigate PI-related problems to enable in-depth understanding, theoretical definition and emphatic measurement of PI concepts.

\section{Thematic analysis}

As illustrated in Figure 3, the largest proportion of the PI literature constitutes themes related to business models (BM); transit centers (TC); seamless, secure, and confidential data exchange (DE); and cooperation models (CM). The vehicle usage optimization (VO), modular containers (MC), and legal models (LF) themes account for a much smaller percentage of the PI literature. Although other themes emerged, their percentages were negligible (see Figure A4 in Appendix 1).

Business models were the most prominent theme-with 43 publications, representing 22 percent of the PI literature-and provide a well-grounded basis for future PI contributions. The first publication discussing the PI and potential business models dates back to 2010, and the numbers have subsequently increased, peaking with 10 publications in 2016. One stream of publications on this theme laid the foundation for the development of PI concepts. Ballot et al. (2010) proposed the concept of interconnected logistics networks, which Montreuil et al. (2012) built upon to conceptualize the main PI components (e.g. hubs, containers and vehicles) and develop a framework for business model innovations enabled by the PI. Montreuil et al. (2013a) also conceptualized additional aspects of the PI, such as the open global logistics system, universal interconnectivity, encapsulation, standard smart interfaces, standard collaborative protocols and innovation-driven SC models. Oktaei et al. (2014) developed a business model for PI hubs that defines customer segments, value propositions, channels, customer relationships, revenue streams, cost structures, key resources, partners and activities, which could all be used to transform existing SCs.

With the increase in the number of publications on this theme, the contributions have become more focused. Hambleton and Mannix (2015) and Chakroun et al. (2016) envisioned the implementation of the PI in Mozambique and Casablanca, respectively, by contextualizing the existing business models for PI hubs. Schoen et al. (2016) proposed a PI-enabled automation of the blood SC in France through a process-mining approach. Zhong et al. (2015) developed a PI-enabled building information-modeling platform for the construction of prefabricated housing in Hong Kong. Treiblmaier (2019) attempted to integrate the PI with Blockchain into a theory-driven framework that aims to maximize PI's 
PI as a new

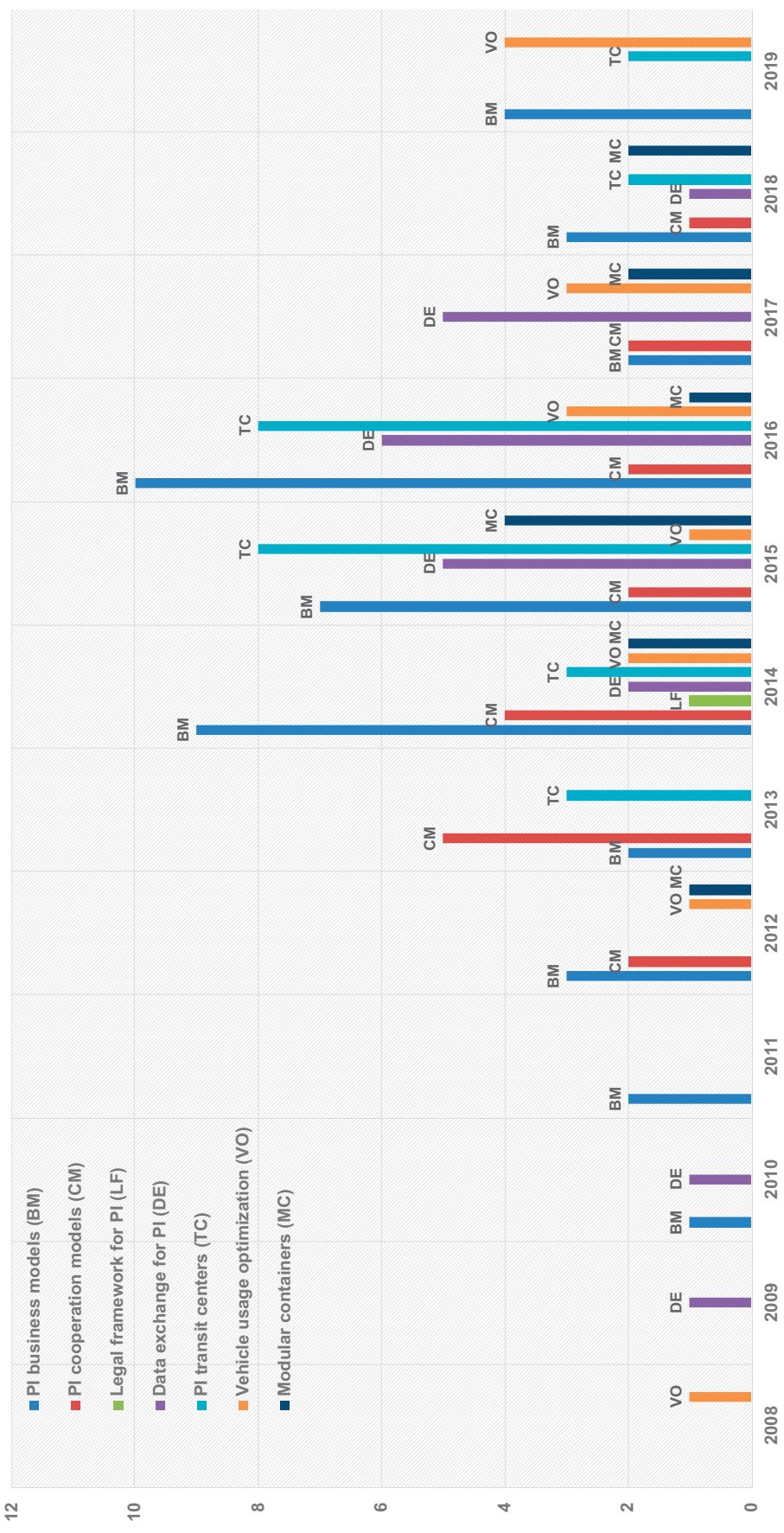

supply chain

paradigm

251

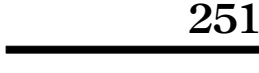


IJLM

31,2

triple bottom-line sustainability-namely, its simultaneous focus on economic, environmental and social goals.

The transit centers theme, which is addressed next in the PI literature, accounts for 14 percent of the literature, with 26 publications. Its contributions commenced in 2013 and increased in the subsequent years. Early research on this theme, namely by Ballot et al. (2013) and Montreuil et al. (2013b), proposed proofs of concept for the functional designs of unimodal (road-based) and multimodal (road-to-rail) PI hubs. Pan et al. (2013) measured the impact of PI hub operations on stock and inventory levels to highlight the benefits associated with the implementation of PI-enabled open logistics networks. Further contributions to this theme evaluate and optimize certain aspects of the early conceptualization of PI hubs and optimized PI container allocation for the functional design of the multimodal (road-to-rail) PI hub proposed by Ballot et al. (2013). Pach et al. (2014) assessed and optimized PI container routing for the functional design of the unimodal (road-based) PI hub proposed by Montreuil et al. (2013b).

Contributions to this theme have become less general and more context-specific, and now include fewer references to prior conceptualizations of PI hubs. Qiao et al. (2016a, b) proposed a dynamic pricing model to the bid prices and profits of carriers in PI hubs. Hao and Gue (2016) designed and assessed a control scheme for a two-sided, grid-based rail-to-rail PI hub. Cheng et al. (2016) developed a multi-attribute double auction system for perishable SC trading in PI hubs. Kong et al. (2016) proposed a scheduling solution for the trolley loading and auction trading of perishable goods in PI hubs. Chargui et al. (2019) developed a multiagent-based model for the truck scheduling and container assignment and grouping in a road-to-rail PI hub.

With 21 publications, the seamless, secure and confidential data exchange theme covers 11 percent of the PI literature. The early contributions regarding this theme conceptualize the data exchange foundations for the PI. Hakimi et al. (2009) conceptualized the basic elements for supply web technology, including (1) a supply web database system that standardizes and unifies web members' supply data and (2) a set of business intelligence tools. Building on this conceptualization, Hakimi et al. (2010) proposed a multi-agent, distributed and modular supply web simulation platform for visualizing, monitoring, mining and assessing data exchanges.

As the number of contributions regarding this theme increased, one stream of research began to address the data exchange in PI components such as hubs and containers. Tretola and Verdino (2014) proposed a conceptual approach with the description of a data model for the modular logistics and a processes overview for a PI-enabled interconnected logistics network. Further building on their study, Tretola and Verdino (2015) introduced high-level ICT (Information and Communication Technologies) architecture for PI-enabled IT systems for an intelligent network management system at PI hubs. Correspondingly, Tretola et al. (2015) proposed an approach for handling information about modular boxes in an interconnected logistics scenario using a canonical data model, which is an enterprise application integration pattern based on the MODULUSHCA common data model for the encapsulation of data in PI containers.

Another research stream focuses on the application of data exchange standards to different contexts. Le Roch et al. (2015) contextualized the EPC global standards and related technologies to the management of reusable pallets in PI-enabled interconnected logistics networks. Zhong et al. (2016) proposed a PI-enabled manufacturing system for intelligent workshop production, which uses RFID and wireless communication networks for the collection and synchronization of real-time data. Some contributions to this theme further improve PI-related data exchange practices and standards. Zhang et al. (2016) developed a design for a PI-based recyclable, world-standard smart green box for encapsulating customer orders. Chen et al. (2018) proposed and assessed a PI-enabled building information modeling 
system (PI-BIMS) that integrates Auto-ID technologies, BIM and cloud computing. PI-BIMS enables collecting, transmitting, processing and visualizing the real-time project information across the project processes.

With 18 publications, the cooperation models theme accounts for 9 percent of the PI literature. The majority of the contributions regarding this theme address the conceptualization and assessment of cooperation rules, practices and tools for horizontal collaboration in PI-enabled interconnected logistics networks. Verstrepen and Jacobs (2012) conceptualized and assessed an orchestrated horizontal collaboration for road bundling between multiple shippers (i.e. JSP and Hammerwerk) to determine its efficiency, sustainability and effectiveness. Using data from consumer-packed-goods SCs, Meller and Ellis (2012) measured the impact of PI-enabled shared-asset logistics network on the distances that foods travel as they flow through the SC, as well as the inventory held at various points along the SC. Jacobs et al. $(2013,2014)$ conceptualized the development and management of a horizontal collaboration community for fresh and chilled retail distribution between two fast-moving consumer goods shippers (i.e. Nestlé and PepsiCo), a logistics service provider (i.e. STEF) and a neutral trustee (i.e. TRI-VIZOR). Cruijssen et al. (2014) developed and piloted a toolbox and business models for horizontal collaboration within the PI's open logistics networks.

With 15 publications, the vehicle usage optimization theme represents 8 percent of the PI literature. Contributions to this theme focus on the design and assessment of PI-enabled solutions for the optimization of vehicle usage. Othmane et al. (2014) modeled a centralized transportation auction mechanism for PI networks to address the problem of determining winning PI carriers (known as the Winner Determination Problem) based not only on bid prices but also on the reputation of PI carriers in open logistics networks. Hofman (2016) proposed an alternative solution based on a resource paradigm for the optimization of PI carriers' capacity utilization. Ji et al. (2019) proposed of a mixed-integer linear programming formulation for addressing the problem that combines an integrated production-inventorydistribution decision with PI.

With 12 publications, the modular container theme accounts for 6 percent of the PI literature and primarily addresses the conceptualization, assessment and optimization of PI containers. Montreuil et al. (2014) proposed a three-tier modular design for PI containers: T containers, $\mathrm{H}$ containers and $\mathrm{P}$ containers. Landschützer et al. (2015) developed a methodological engineering process for the first approach used to develop modular and multifunctional PI containers. Tran-Dang et al. $(2015,2016)$ introduced a system to automatically generate and maintain a virtual 3D layout reflecting the spatial distribution of PI containers. Krogsgaard et al. (2018) developed a new approach for solving the "liner shipping network" design problem that is based on the PI-enabled distributed multisegment flow.

The legal system for the PI theme is in the early development stages, with one publication, accounting for only 0.5 percent of the PI literature. Biermasz and Louws (2014) discussed the necessary changes made to the legal framework to support horizontal collaboration initiatives in Europe, such as the CO3 Project. Figure 4 illustrates the trends of the main PI themes over the years.

\section{Methodological analysis}

As shown in Figure 5, solution design research $(\mathrm{AS}+\mathrm{S})$, conceptual research $(\mathrm{C})$ and assessment research (A) constitute the largest percentage of the PI literature, while valuation research $(\mathrm{V})$ and literature research $(\mathrm{L})$ represent only a small percentage. There are other methods in the PI literature; however, they do not account for a significant percentage (see Figure A5 in Appendix 1).
PI as a new supply chain paradigm 
IJLM
31,2

254

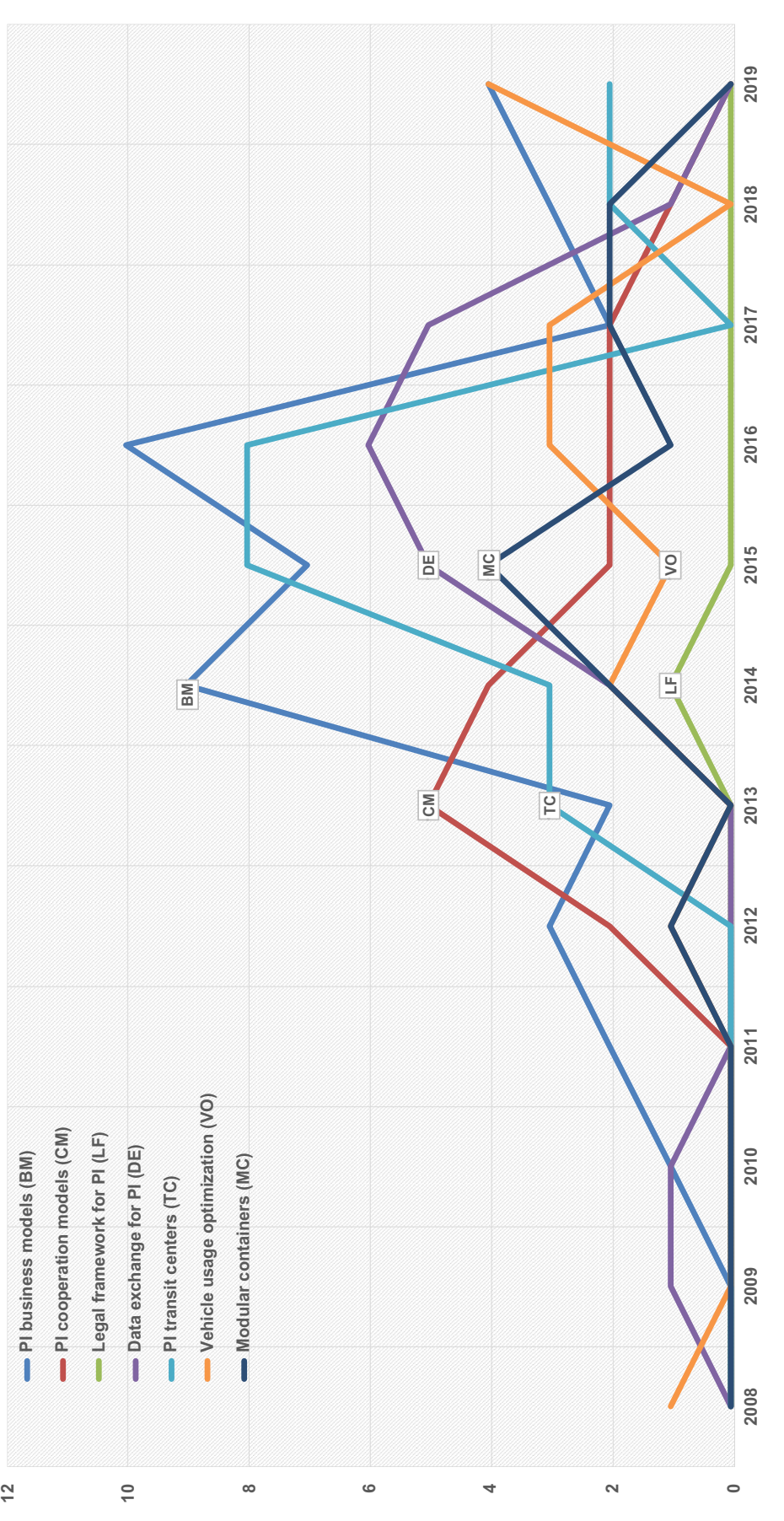

Figure 4.

Change and Trends of main PI themes over years 


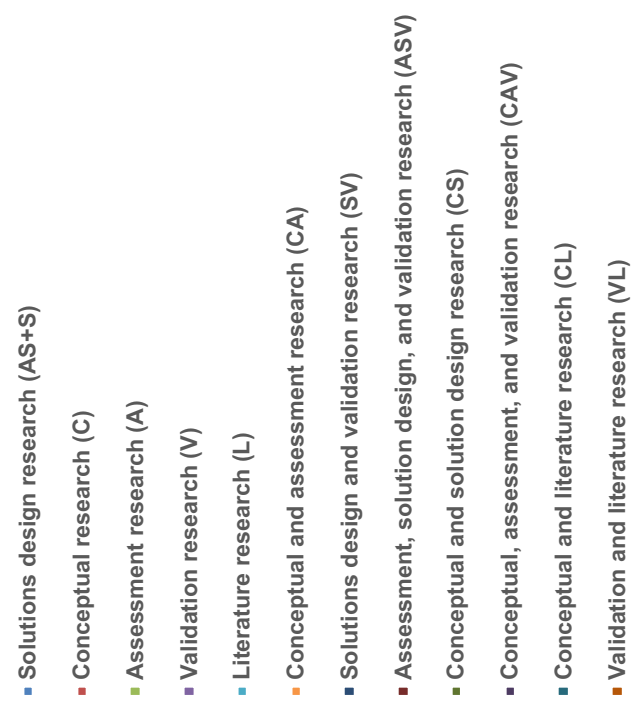

PI as a new supply chain paradigm

255

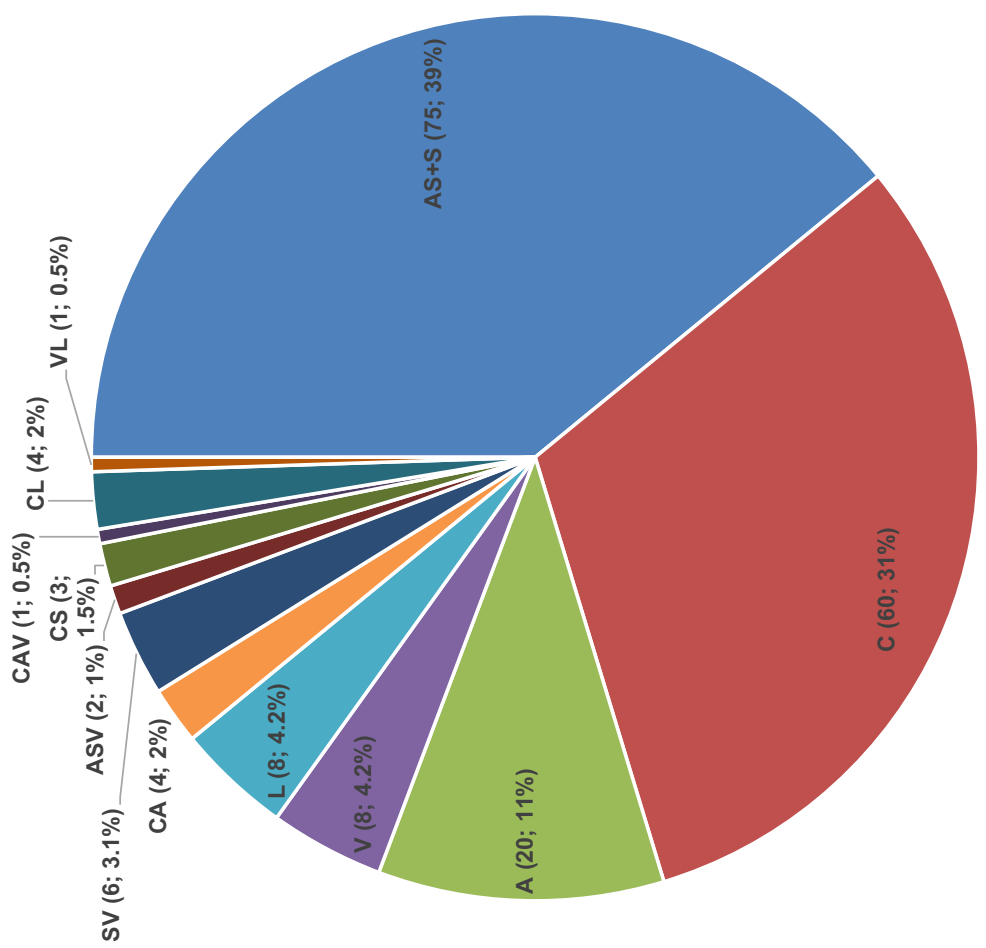

Figure 5. Methods in the PI literature in percentages 
IJLM

31,2

With 60 publications, the conceptual research category accounts for 31 percent of the PI literature. Contributions on the conceptualization of the PI started as early as 2009 and steadily increased in number over the years before reaching its peak at 2014 with 10 publications. This research stream focuses on defining and refining fundamental PC concepts, such as business models, hubs, containers, vehicles and carriers. This research category also conceptualizes the PI implementation in specific industries (e.g. FMCG), countries (e.g. France) and regions (e.g. European Union). The PI concept was initially introduced by Montreuil et al. (2010), Ballot et al. (2010), Lounès and Montreuil (2011), and Montreuil (2011, 2012). After establishing the foundations of the PI, Montreuil et al. (2013a), Ballot et al. (2014) and Sarraj et al. (2014b) further conceptualized and refined certain PI concepts and foundations, such as the benefits and challenges associated with its implementation.

Over time, the conceptual research has become more focused, addressing specific contexts that are relevant to the PI. Montreuil et al. (2012) proposed a model for open logistics interconnection, which is based on the open system interconnection model. Crainic and Montreuil (2015) applied the PI concept to the context of city logistics by proposing hyperconnected city logistics. Lin and Cheng (2016) conceptualized and implemented a PIenabled platform for an automatic product identification system, product-production tracking system, finished product scan-and-pack system and electronic shelf system in the solar cell industry. Wang et al. (2016) conceptualized decentralized production scheduling (i.e. initiative production scheduling) for a PI manufacturing system. Puskás and Bohács (2019) examined the applicability of Industry 4.0 tools — such as cloud, mobile technology, Big Data and analytics, decentralization, and simulation - to improve the PI components including PI container, PI hub, PI sorter, hub-and-spoke transport and others.

With 75 publications, the solution design research category represents 39 percent of the PI literature. This research preceded the conceptual research and generated notable contributions from 2014 onwards. The solution design research category focuses first on designing and engineering and then on testing methodologies and technologies for the implementation of the PI in certain industries, countries or regions. This category also identifies the barriers which prevent an industry, country or region from adopting the PI, and then designs and develops solutions to facilitate the implementation of the PI.

This research category has two streams focusing on the following areas: 1) designing the key PI components and 2) developing methodologies and models for PI-enabled planning and operational decisions in interconnected logistics and SC networks. In the first research stream, a decomposition-based approach was developed for selecting standardized modular containers, which addresses PI container sizes and dimensions. Gazzard and Montreuil (2015) developed a functional design for PI handing modular containers. Landschützer et al. (2015) addressed PI container requirements and engineering design in terms of sizing (i.e. methods of selecting container sizes depending on the dimensions of goods), design (i.e. key functionality of PI containers) and loading (i.e. conditions for loading). Sallez et al. (2015b, 2016) developed a descriptive framework for the multilayered activeness that enables embedded static or dynamic data, measurement via sensors on shells and skeletons, interaction with other PI containers, measurement of external conditions via sensors and PI management system interaction and intelligence that provides identification, traceability/ tracking, integrity and confidentiality of PI containers. Montreuil et al. (2014) proposed a three-tiered modular design for three types of PI containers, namely transport (T), handling (H) and packaging (P) containers. Ballot et al. (2012, 2013), Meller et al. (2012), Montreuil et al. (2013b), and Oktaei et al. (2014) developed functional designs for road-to-rail bimodal hubs, road-based unimodal cross-docking hubs and business models for transit centers.

In the second research stream, the focus is on developing PI-enabled planning models. Xu et al. (2013) designed a contribution-and-power weighted value (CPWV) sharing mechanism 
based on game theoretic solutions for transportation service procurement in PI-enabled horizontal collaborations. Sallez et al. (2015a) proposed a hybrid PI hub control architecture with an efficient reactive PI container routing approach to accommodate the short-term external or internal perturbations in a PI cross-docking hub. Qiao et al. (2016a, b) developed a dynamic pricing model based on an auction mechanism for optimizing transport service providers' bid prices in PI hubs. Chen et al. (2017) and Pan et al. (2015a) developed a PI-enabled solution for collecting e-commerce goods from final consumption points and returning them to retailers, which delivers the returned goods and passengers in an integrated way by leveraging the extra loading capacity and constant mobility of citywide taxis. Pan et al. (2015b) designed and assessed an inventory-control model for PI-enabled interconnected logistics networks to reduce the system's inventory level and the total logistics costs. Yang et al. (2015a, b) proposed an inventory-control model based on a PI-enabled interconnected logistics services for the FMCG sector. This model was then further assessed, in terms of resilience, by Yang et al. (2016). Naccache et al. (2014) conducted a multi-agent simulationbased assessment of PI-enabled interconnected e-commerce distribution and its implication for inventory levels.

With 20 publications, the assessment research category accounts for 11 percent of the PI literature. After addressing the conceptualization of the PI, the literature assessed the proposed PI concepts. This research stream focuses on measuring the PI's economic, environmental and societal impacts and its sustainability improvements using analytical, optimization or simulation modeling experiments. Hakimi et al. (2012) presented the first comprehensive simulation experiment that assessed the PI's impact in terms of performance and economic, environmental and social efficiency in France. Using a simulation experiment, Sarraj et al. (2014a) conducted a study that measured the transportation efficiency and sustainability improvements of PI-enabled path routing for containers and the minimization of transportation means in the FMCG sector in France. Using analytical modeling, Meller et al. (2012) assessed the impacts of the PI in terms of increased profit margin and reduced environmental footprints in SCs in the United States. Sohrabi and Montreuil (2011) conducted an optimization-based assessment to evaluate the potential economic gain from a PI-enabled interconnected distribution system.

With only eight publications, the validation research category accounts for 4.2 percent of the PI literature; it is, thus, likely in its nascent stage. This research focuses on conducting case studies, field pilot studies, virtual pilot studies and living labs for the implementation of the PI in real-world projects, which informs the PI implementation roadmap. Hambleton and Mannix (2014) drew parallels between the PI initiative and the 3D supply networks project, which both aim to 1) reduce the economic order quantities from a pallet to a case, 2) move the inventory closer to customers, 3) reduce the highway miles traveled, and 4) improve truck utilization. Hambleton and Mannix (2015) presented the case study of the Sunshine Nut Company, which aims to transform the lives of Mozambicans, much as the PI will likely transform global logistics. Through a partnership with ES3, Sunshine Nut is leveraging ES3's PI hub facility for warehousing and distribution, which in turn is driving financial, environmental, societal and transformational change. ALICE (2014a, 2014b, 2014c, 2015a, 2015b, 2015c) reported on the PI implementation roadmap for the 2016-2017 period in terms of urban freight, SC coordination and collaboration, corridors and hubs, IS for interconnected logistics and the sustainability, safety, and security of SCs.

The literature research category accounts for 4.2 percent of the PI literature (eight publications). It focuses on reviewing and summarizing the extant PI literature and outlining future research directions. Treiblmaier et al. (2016) reviewed the existing PI literature in the form of conference proceedings, reports, books and journals, and then called on researchers to explore the synergies between SCM and the PI. Sternberg and Norrman (2017) reviewed and analyzed the existing literature on the PI using Iacovou et al. (1995) theoretical framework for
PI as a new supply chain paradigm 
IJLM
31,2

258

Figure 6.

Change and trends of main methods in the PI literature over years

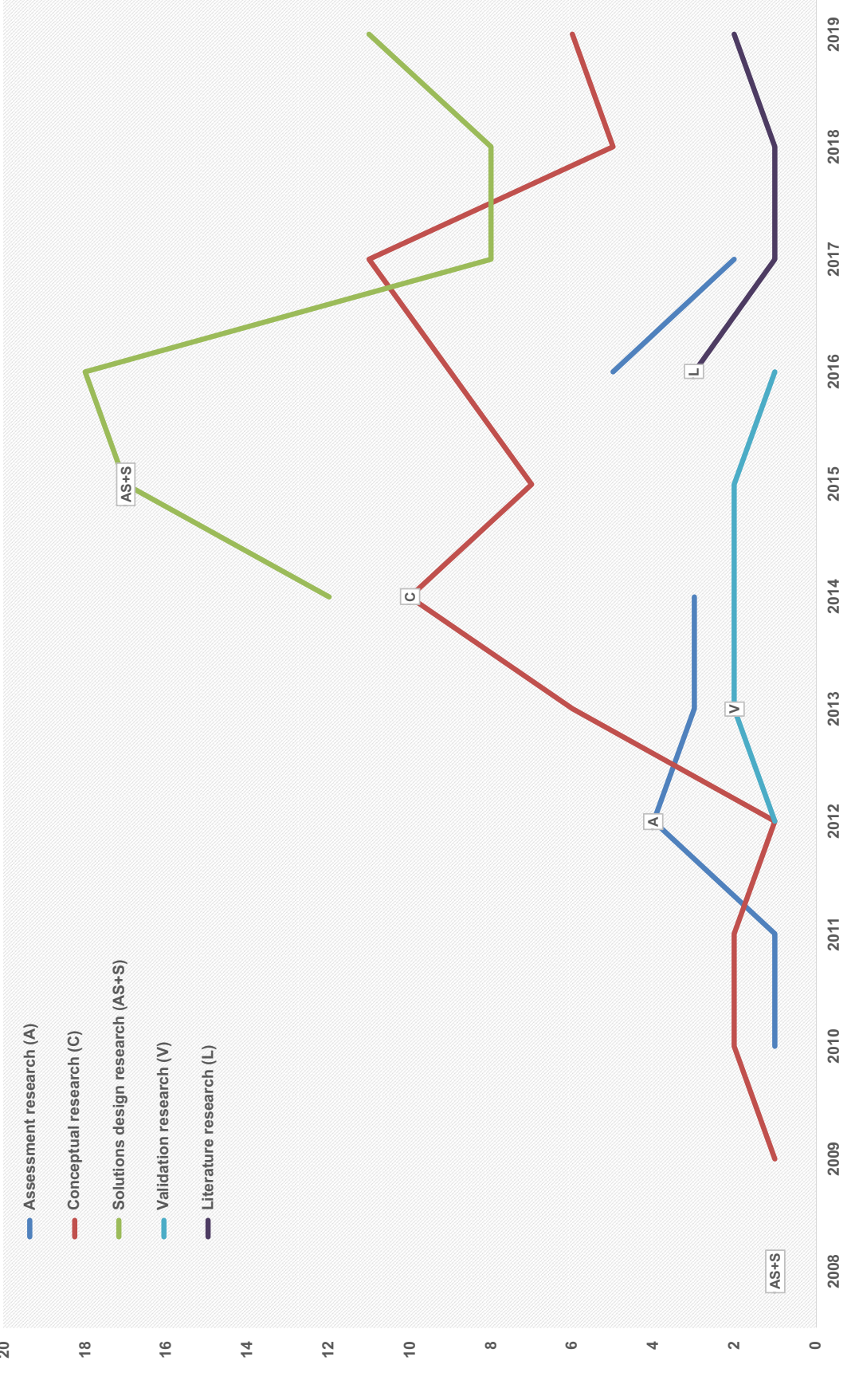


the technology adoption of firms in an interorganizational context. Figure 6 illustrates the trends of the main PI methods over the years.

\section{Discussion}

The $\mathrm{PI}$ is receiving increasing attention from academics and practitioners, who view this new paradigm as being a disruptive innovation having the potential to significantly disrupt existing logistics and SC practices. This has resulted in a steadily growing number of publications, dedicated conferences and substantial funding opportunities. Consequently, it is important to better understand the emerging themes and methods in the PI literature, which should help logistics and SCM researchers identify future directions for PI research regarding innovation in SC models and strategies. Accordingly, we conducted a SLR to examine the evolution of the PI literature by identifying its main themes and methods.

\section{The evolution of the PI literature}

Based on an analysis of the underlying literature and the change in research focus over time, we labeled the three stages that describe the evolution of the PI literature as "incubation," "exploration" and "expansion." In the incubation stage (2008-2011), the PI was not explicitly defined, except in a few conceptual publications. This stage includes eight publications that attempt to envision the PI concept and its impact logistics and SCM. Most of the publications in this stage are purely conceptual and attempt to conceptualize the PI without any significant contributions to the PI concepts. The exploration stage (2012-2014), which includes 51 publications, focuses primarily on defining PI principles and its fundamental concepts, such as business models, hubs, containers, vehicles and carriers. The majority of the publications in this stage focus on conceptualizing and assessing the proof-of-concepts for the PI concepts. In the expansion stage (2015-2019) with 133 publications, researchers' efforts have switched from pure conceptualization to the investigation of specific aspects of the PI or the assessment of its proposed concepts. The majority of the publications in this stage focus on designing, engineering and testing methodologies and technologies for the implementation of the PI in certain industries, countries or regions.

\section{A PI-Based framework}

Based on our thematic analysis, we developed a framework that outlines the main PI themes, including the facilitators and barriers, emerging from the literature (see Table AII in Appendix 1). Overall, these themes present different layers of potentially disruptive developments, ranging from creating basic components such as standardized containers to overarching business models, all of which lead to greater innovation in logistics and SC strategies. In the extant literature, considerable effort seems to have been invested in establishing these themes as central components of the PI around which practical solutions can be developed. Figure 7 shows the themes in a hierarchical order in which similar to the Internet protocol TCP/IP, each layer builds on its previous one. To retain the abstract nature of this framework, we did not include specific technologies. The themes in our PI-based framework fit each other and together they can exert their full potential. This does not mean, however, that these potentially disruptive developments cannot function independent of each other. It rather suggests that innovation can happen at different layers simultaneously, subsequently combining into a one holistic and comprehensive concept that drives disruptive $\mathrm{SC}$ strategies.

First, modular containers research conceptualizes shared, modular, sustainable, robust, lightweight and scalable PI containers. Using smart tags, including RFID and GPS technologies, PI containers collect and store logistics and SC information, which ensures container identification, integrity, routing, conditioning, traceability and security in
PI as a new supply chain paradigm

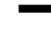




\section{IJLM \\ 31,2}

\section{0}

Figure 7.

PI facilitators, themes and detractors

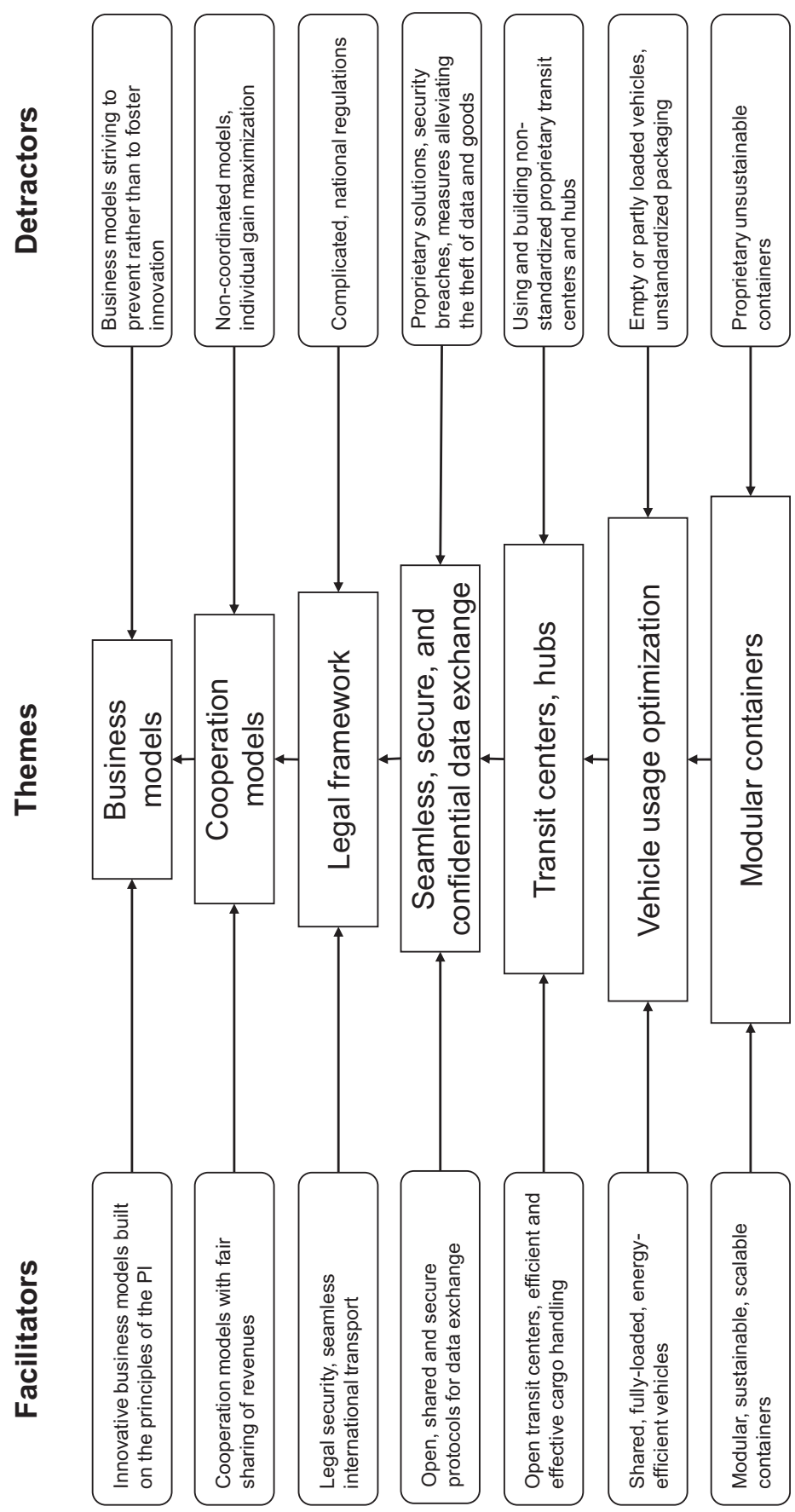


interconnected logistics network. Thus, PI containers provide the foundation for sophisticated SCM that is characterized by real-time logistics and SC data flow and analysis.

Second, vehicle usage optimization research proposes the idea of using shared, fully loaded, energy-efficient PI vehicles with relays, which reduces transportation costs and carbon footprints. Such research attempts to solve the problem of groupage transportation, including consolidation and deconsolidation centers in open-logistics network nodes in which goods are loaded/unloaded in/out of PI containers. Using mixed-integer linear programming models, the objective is to meet the demand of shipment orders belonging to different areas with the final goal of minimizing total costs, exploiting trucks capacity and reducing empty trips.

Third, transit centers research encourages the move from non-standardized proprietary transit centers to modern and open PI transit centers for efficient and effective cargo handling. Using coordination algorithms for matching demand and supply, the mission of PI transit centers is to efficiently and sustainably transfer PI-trailers from one truck to another. This is done specifically for two purposes: (1) to enable PI containers to move from their origin to its destination, facilitating delivery within the delivery time window and (2) to enable trucks to pick-up a PI container that will put the driver closer to the target destination at the end of a workday.

Fourth, the seamless, secure and confidential data exchange research defines a set of open, shared and secure protocols for data exchange in PI-enabled open logistics networks, which restrict access to data on goods and information about delivery status. Such research leverages multiple data models, including canonical data and enterprise application integrations, to define a common set of data and information for information exchange and interoperability between participants in open logistics network.

Fifth, legal framework research aims to synchronize the incompatible legal environments associated with different countries to provide legal security and seamless international transport. This, legal framework is especially useful for synchronizing the legal environment amongst the 28 European Union counties that have relatively disparate legal systems.

Sixth, the cooperation models research attempts to redefine the existing practices for revenue sharing among different stakeholders in the new PI-enabled business models, such as PI hub holders and PI movers. This breakthrough in the cooperation practices should facilitate openness among logistics and SC partners.

Seventh, business model research strives to foster innovation in logistics practices and SCs by using the 13 principles of the PI. SC innovation is critical for companies to survive global competition and the PI can be fundamental to this innovation. The PI provides a new paradigm to encourage the development of innovative ideas that are based on PI to directly impact and improve the effectiveness and efficiency of current logistics and supply chain practices both in domestic and in global applications.

\section{Future research directions and approaches}

We further analyzed the main PI themes in relation to the methods to provide directions for future research and theory development. We sought additional research opportunities within the context of the existing PI publications in terms of themes and methods. A growing and relatively new research area can become more robust as the variety of research methods and areas increases over time. Figure 8 illustrates the main PI themes and their corresponding methods.

It is important to emphasize that some areas are under-researched, and some methods are underutilized for numerous reasons. There may be a lack of data, the research questions may be uninteresting or the research methods may be imprecise. However, by identifying the research areas with a high number of contributions and commonly used methods, we showed
PI as a new supply chain paradigm 
IJLM

31,2

\section{2}

Figure 8.

Main PI themes with corresponding methods

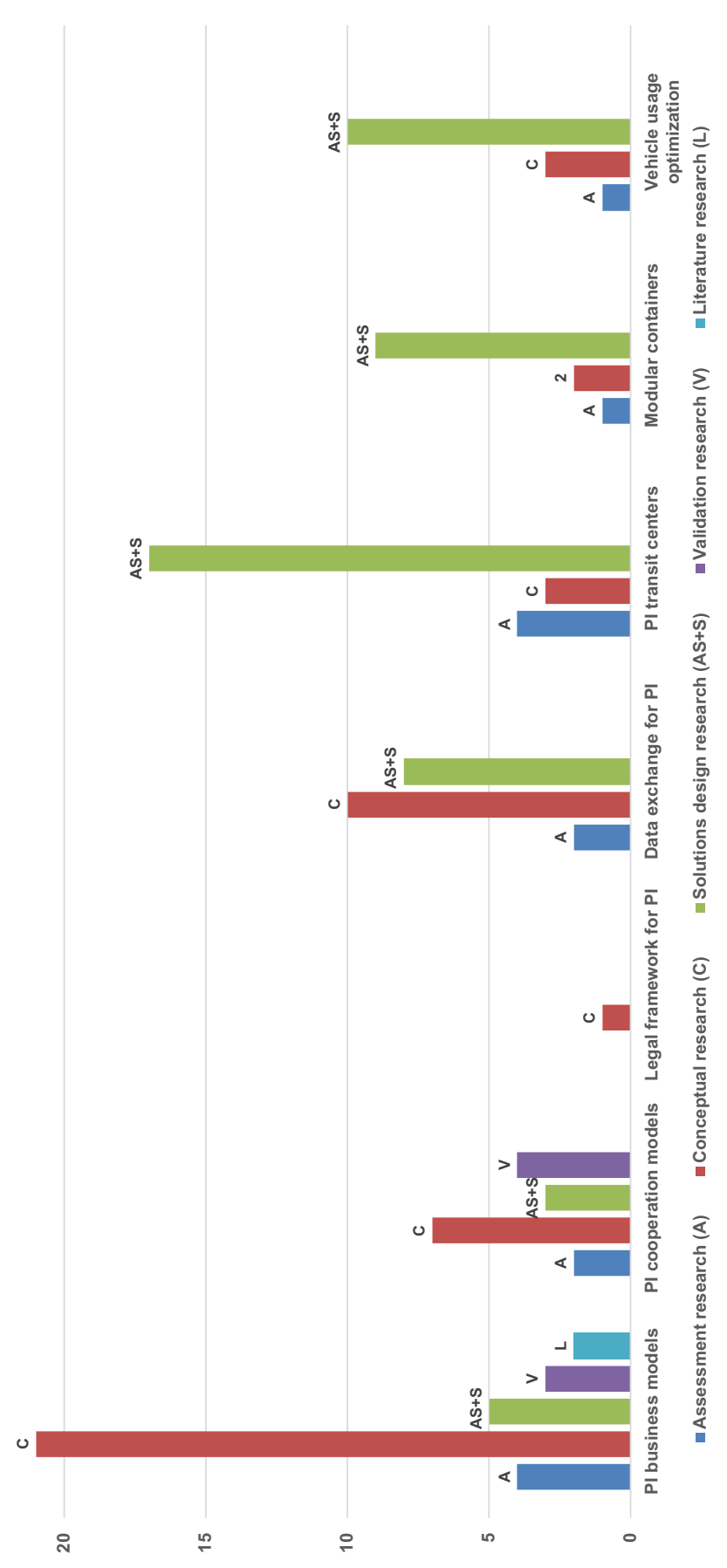


the potential gaps in PI research. Following the same logic, research areas with a low number of contributions and underutilized research methods might help researchers identify new areas to research. Based on our analysis of the PI literature, there are five major takeaways that can be considered for future PI research.

First, business models, transit centers, seamless data exchange and cooperation models are the most researched PI themes. Based on our analysis, conceptual, solution design and assessment are the most commonly used methods in the main PI themes. Hence, there is an opportunity for future PI research to be conducted on the outlined main themes using the following research methods:

(1) Conceptualization of multimodal road-to-sea- and rail-to-sea PI hubs.

(2) Validation of unimodal (road-based) and multimodal (road-to-rail) PI hubs within a real-life case study.

(3) Validation of the proposed protocols and systems for data exchange in PI-enabled open logistics networks.

Second, vehicle usage optimization, modular containers and legal models are the least researched themes, and within these, solution design is the most commonly deployed method. Hence, there is an opportunity for future PI research on these under-researched themes using the following research methods:

(1) Validation of the proposed design and types of PI containers within a real-life case study.

(2) Validation of the proposed auction mechanism and optimization of capacity utilization for PI carriers in PI hubs within a real-life case study.

(3) Conceptualization of a legal framework for trans-Atlantic horizontal collaborations such as between the United States and the European Union.

(4) Assessment, optimization and validation of the proposed legal frameworks for horizontal collaboration within a real-life case study.

Third, one major question arising from our literature review pertains to the right approach to fully realizing the vision of the PI, in which a comparison with the digital Internet might be helpful. Due to its layered nature, it is possible to improve specific aspects of the Internet without disrupting the functioning of the whole system, which has been done by individual researchers or organizations. For example, the TCP/IP protocol was developed in 1982, HyperText Markup Language (HTML) in 1990 and Mosaic (First Web Browser) in 1993 all by independent researchers and all improved specific aspects of the Internet that are critical to its current operations. Likewise, researchers can improve specific aspects or themes within the PI without disrupting the functioning of the overall system, assuming that the interfaces remain operable. For example, modular containers need to be designed to optimize their use, to efficiently and effectively use cargo handling in transit centers and hubs. Similarly, seamless, secure and confidential data exchange supports the physical flow of goods, and legal frameworks that are needed to create trust between the parties involved.

Fourth, most PI technologies have not fully matured and thus are under continual development. More research is needed to develop, test and assess the influence of these technologies on PI-enabled open-logistics networks. Academics that tend to focus on the theoretical level of the PI can learn from practitioners who can test the theoretical models in real-world applications. Likewise, practitioners who mainly focus on the development of the PI to either reduce costs or increase revenue can learn from academics to find new theoretical

PI as a new supply chain paradigm . 
IJLM

31,2

tools to implement the PI successfully. Close collaboration between practitioners and academics thus can help to yield valuable insights on how to best achieve the goals of the PI.

The fifth insight we gained from the literature review is the many opportunities for further theory development related to PI research. Few of the many PI publications explicitly incorporated theory. For those papers that did include theories we found the theories were only slightly modified for the unique PI context. Many of the theories used (e.g. agency theory, auction theory, game theory) were not well-contextualized to the PI assumptions and outcomes. For example, game theory and agency theory focus on well-researched outcomes (e.g. reduction in costs, increased in efficacy, increases in profits); however, they do not consider some of the outcomes that make the PI compelling (e.g. sustainability, interconnectedness, standardization, encapsulation). Such a lack of PI contextualization impedes meaningful PI empirical research. Hence, there is an urgent need for a carefully contextualized theoretical foundation that will accommodate the unique characteristics of the PI and lead to greater insight and even more successful outcomes. The application of theorybased research to gain further insights is not bound to a specific layer of our framework and it is therefore up to future researchers to identify and develop existing and new theories that are suitable to explore, explain and predict phenomena at different levels and between them.

As an initial start to developing theories that are more contextualized for the PI, we recommend the model we developed in Figure 7 . This model can be viewed as a comprehensive framework that covers many of the relevant themes of SCM in a layered manner; thus, it can also help researchers identify relevant theories at each layer. As an illustration, interconnectivity, openness, and secure data exchange incorporate informationprocessing theory. From an economic perspective principal-agent theory, transaction cost analysis, resource-based view and network theory can help researchers better understand how the PI influences structural and managerial aspects of SCs. Practical suggestions on how to derive specific research questions based on theory can be found in Halldórsson et al. (2007) for third-party logistics and new-product development as well as in Treiblmaier (2018) for Blockchain. These studies apply the aforementioned managerial and structural theories to answer the questions of how to structure a SC. It is our hope that future research would consider this framework to either develop new theories specifically for the PI or take existing theory and incorporate PI context factors such as sustainability, interconnectedness, standardization and encapsulation.

\section{Contributions to the PI literature}

The contribution of our SLR to the progress of the PI research is threefold. First, we demonstrate the evolution of the PI literature by analyzing its key themes and methods. By doing this, we illustrate how this concept strongly relates to logistics and SCM research and connects academia with industry. This SLR not only includes many conference papers and industry reports but also a steadily growing number of quality journal papers, thereby illustrating the rapidly increasing interest in the PI concept. We show that a multitude of interesting ideas and applications exist to create more effective, efficient and sustainable procedures for logistics and SCM, all of which may lead to a radical shift in existing SC models and strategies.

Second, we identify the main themes of the PI that help us to outline unanswered questions. These themes also serve as a roadmap for future research and, more specifically, meaningful research that is contextualized to the PI. The proposed PI-themed framework should help PI researchers find relevant under-researched topics and under-utilized research methods. This will directly improve research on the PI and has the potential to transform logistics and SCM research and practice. Furthermore, categorizing the literature according to the themes and methods allows for a structured comparison between what has already been achieved in PI literature and where research gaps exist. This natural evolution of the PI literature is likely to be 
more effective just like how the digital Internet evolved than using a top-down approach to define how the respective characteristics of a full-fledged PI should look. However, a top-down approach can be another avenue to pursue in future PI-related research.

Third, our PI-themed framework is based on the extant literature and introduces PI facilitators and barriers. It can thus help researchers to identify the relationships between the drivers, processes and outcomes to enhance our understanding of the PI phenomenon. There is also a possibility that the PI can be viewed as more than just a disruptive innovation or radical shift for existing logistics and SC processes. Rather, the PI can be regarded as an innovation platform like the digital Internet that provided a foundation for many new disruptive innovations. In the same way, the PI has the potential to become an innovation platform that provides an opportunity for the development of radically new products or services for the logistics and SC industry, leading to significant improvements in the environment, economy and society.

\section{Managerial implications}

The implications for managers are fourfold. First, despite academics' steadily growing interest in the PI, we have shown that the vast majority of the existing literature is still directed toward practitioners and academics who work in close cooperation with the industry. PI business models, PI hubs and interorganizational data exchange are areas of utmost importance for organizations that are seeking to improve their current logistics and SCM practices. Thus, a clear structure, which includes the categorization of the relevant themes and methods, as provided in this PI literature review, should help guide research activities in the industry. This enables managers to better understand the full scope of the PI and the respective contributions of technological advancements.

Second, the widespread diffusion of the PI necessitates that managers fully understand the subtleties of this comprehensive and complex concept. In this SLR, we have, therefore, broken down the PI into its main themes and methods. This will enable managers to better understand the "big picture" as well as the various research streams that can be derived directly from it. A shared understanding of the PI will, thus, help managers to launch their own future research endeavors and engage in interorganizational cooperation when undertaking PI-related projects. Ideally, this will result in increased SC efficiencies and substantial cost savings.

Third, various funding opportunities and international networks are available that foster interorganizational cooperation to achieve synergies that benefit all network participants. By the same token the PI is a concept that necessitates cooperation beyond organizational boundaries and is dependent on the support of funding agencies and supranational entities. Funding at the national and international levels should be dedicated to those PI projects that can emphasize the practical impact of their project goals. These opportunities allow organizations to participate in research projects and benefit from knowledge sharing.

Fourth, our PI-based framework outlines the key facilitators and barriers that have direct influence on the adoption of the PI. These facilitators and barriers can serve as valuable implication for policymakers and practitioners who have interest in a partial or full adoption some PI concepts. For example, policymakers should consider supporting projects on the public administration and policy side, which encourage logistics and SCM practitioners to change their existing behaviors and move to more interconnected logistics services in urban areas. More specifically, municipalities can contribute to this transition by setting explicit sustainable urban logistics practices, including zero-emissions logistics and limitations on the presence and frequency of delivery trucks in large urban areas, which aim to reduce the impact of logistics activities on city inhabitants by encouraging PI-enabled collaborative city logistics. City municipalities can also amend the policies for public procurements to favor
PI as a new supply chain paradigm 
IJLM

31,2

zero-emissions and energy neutral suppliers, which can help trigger change in current logistics practices.

\section{Limitations and conclusions}

Several limitations exist with our SLR, many of which can lead to further research opportunities. First, although we did our best to make the search, selection and validation processes as transparent as possible, a certain amount of subjectivity exists when it comes to the publications' classifications. Several publications, for example, used a mixed-method approach and thus we needed to identify the predominant method. Although this was done in a research team for increased validity, we acknowledge the subjectivity of this approach.

Second, this SLR reflects the state-of-the-art of PI research and does not attempt to specify any priority of specific research topics, whose importance should be determined by the several research groups in academia and practice groups in industry. Examples for the latter include several working groups of the European Union which influence the relative attention given to specific topics by allocating funding. Given the innovative and dynamic changes that are rapidly occurring with PI practice and research, we expect these preferences to change and evolve over time.

Third, the PI is a comprehensive framework that is used by practitioners to improve logistics or SCM as a whole. Most research activities focus on specific parts of the framework. However, the increasing popularity of this concept, as is evidenced by a growing number of scholarly publications as well as funding opportunities, makes it necessary to clearly define and operationalize the respective constituents. Presently, no comprehensive theory of the PI exists but this is not very surprising at this relatively early stage of development. Just like there are many theories of SCM that are used to explain specific components of SCM, the PI will also have multiple theories to explain specific components. It is beyond of the scope of this paper to provide a comprehensive theory of the PI but our SLR is an important first step and we believe that a more comprehensive PI theory will constitute a valuable future research contribution.

Finally, the inclusion of large number of publications outside of top-tier logistics and SCM journals could introduce potential topic biases. However, we felt this inclusion criterion was necessary because of the novelty and emerging nature of the PI. Namely, we decided to include such a broad scope to capture emerging and innovative research that has not had time to be published in elite logistics and SCM journals.

This SLR has demonstrated that the PI is a holistic logistics and SCM concept that addresses many challenges associated with existing logistics and SC models. The growing literature on the PI and its potential to disrupt existing SC and logistics strategies suggests the need for a SLR that assesses the current state of the literature with a view to identifying future research directions and approaches. Accordingly, our SLR categorizes existing PI publications according to themes and methods, depicting the evolution of the PI literature through three stages. Based on the literature analysis, this research proposes a comprehensive framework that structures the PI domain and outlines the future research directions for logistics and SCM researchers. As more PI research is conducted, there are likely to be more applications of the PI, more developments of disruptive innovations that will lead to improvements in productivity, efficiency and sustainability within supply chains that will directly improve the quality of life and society as a whole.

\section{References}

ALICE (2014a), “ALICE: recommendations to H2020 work programs 2016-2017”, available at: http:// www.etp-logistics.eu/wp-content/uploads/2015/07/ALICE-Recomendations-HORIZON2020-WP2016-2017-v141218_DEF-2.pdf (accessed 10 December 2016). 
ALICE (2014b), "Global supply network coordination and collaboration: research and innovation roadmap", available at: http://www.plpt.com.pl/do_pobrania/alice_map_wg4.pdf (accessed 12 August 2016).

ALICE (2014c), "Urban freight: ALICE/ERTRAC Urban mobility WG", available at: http://www. ertrac.org/uploads/documentsearch/id36/ERTRAC_Alice_Urban_Freight.pd (accessed 28 November 2016).

ALICE (2015a), "Corridors, hubs and synchromodality: ETP-ALICE", available at: http://www.etplogistics.eu/wp-content/uploads/2015/08/W26mayo-kopie.pdf (accessed 10 August 2016).

ALICE (2015b), "Information systems for interconnected logistics: ETP-ALICE", available at: http:// www.etp-logistics.eu/?page_id=89 (accessed 10 August 2016).

ALICE (2015c), "Sustainable, safe and secure supply chain: ETP-ALICE Research and innovation roadmap", available at: http://www.etp-logistics.eu/wp-content/uploads/2015/07/W16mayokopie.pdf (accessed 10 July 2016).

Armbrust, M., Fox, A., Griffith, R., Joseph, A.D., Katz, R., Konwinski, A., Lee, G., Patterson, D., Rabkin, A. and Stoica, I. (2010), "A view of cloud computing", Communications of the ACM, Vol. 53 No. 4, pp. 50-58.

Ballot, E., Montreuil, B. and Fontane, D. (2010), "Topology of logistics networks and the potential of a Physical Internet", available at: https://www.picenter.gatech.edu/sites/default/files/topology_pi_ ballot_montreuil_fontane.pdf (accessed 14 October 2019).

Ballot, E., Montreuil, B. and Meller, D.R. (2014), "The network of logistics networks", Paris, France: Direction de L'information Légale et Administrative, available at: http://www. ladocumentationfrancaise.fr/catalogue/9782110098658/index.shtml (accessed 12 August 2016).

Ballot, E., Montreuil, B. and Thivierge, C. (2012), "Functional design of Physical Internet facilities: a roadrail hub", in Montreuil, B., Carrano, A., De Kostner, M.M.R., Gue, K.R., Ogle, M. and Smith, J. (Eds), Progress in Material Handling Research, Material Handling Industry of America, Charlotte, North California.

Ballot, E., Montreuil, B. and Thivierge, C. (2013), "Functional design of Physical Internet facilities: a road-rail hub", CIRRELT, available at: https://www.cirrelt.ca/DocumentsTravail/CIRRELTFSA-2013-14.pdf (accessed 12 August 2016).

Beliën, J. and Forcé, H. (2012), "Supply chain management of blood products: a literature review", European Journal of Operational Research, Vol. 217 No. 1, pp. 1-16.

Biermasz, J. and Louws, M. (2014), "Legal framework transformation", CO3 Project, available at: http:// www.co3-project.eu/wo3/wp-content/uploads/2011/12/CO3-D-2-9-Legal-Framework-excl-contr.august-2014.pdf (accessed 12 August 2016).

Chakroun, A., Abbar, H. and Elaraki, M.T. (2016), "Hyperconnected city logistics and last mile delivery in Casablanca city", 3rd International Physical Internet Conference, June 29-July 1, Georgia Institute of Technology, Atlanta, Georgia.

Chargui, T., Bekrar, A., Reghioui, M. and Trentesaux, D. (2019), "Proposal of a multi-agent model for the sustainable truck scheduling and containers grouping problem in a road-rail Physical Internet hub”, International Journal of Production Research, Vol. 2019, pp. 1-25.

Chen, C., Pan, S., Wang, Z. and Zhong, R.Y. (2017), "Using taxis to collect citywide e-commerce reverse flows: a crowdsourcing solution”, International Journal of Production Research, Vol. 55 No. 7 , pp. 1833-1844.

Chen, K., Xu, G., Xue, F., Zhong, R.Y., Liu, D. and Lu, W. (2018), “A Physical Internet-enabled building information modelling system for prefabricated construction", International Journal of Computer Integrated Manufacturing, Vol. 31 Nos 4-5, pp. 349-361.

Cheng, M., Xu, S.X. and Huang, G.Q. (2016), "Truthful multi-unit multi-attribute double auctions for perishable supply chain trading", Transportation Research Part E: Logistics and Transportation Review, Vol. 93, pp. 21-37.

PI as a new supply chain paradigm 
IJLM

31,2

Christensen, C. (2013), The Innovator's Dilemma: When New Technologies Cause Great Firms to Fail, Harvard Business Review Press, Boston, Massachusetts, MA.

Crainic, T.G. and Montreuil, B. (2015), "Physical Internet enabled interconnected city logistics", Centre Interuniversitaire de Recherche sur les Réseaux d'Entreprise, la Logistique et le Transport (CIRRELT), available at: https:/www.cirrelt.ca/DocumentsTravail/CIRRELT-2015-13.pdf (accessed 19 May 2017).

Cruijssen, F. (2012), "Framework for collaboration (deliverable D2.1)", CO3 Project, available at: http:// www.co3-project.eu/wo3/wp-content/uploads/2011/12/CO3-D-2-1-Framework-for-collaborationfull-report-2.pdf (accessed 12 August 2016).

Cruijssen, F., Van Amelsfort, L., Biermasz, J. and Louws, M. (2014), "Method and tool support for the pilot projects: a CO3 position paper", CO3 Project, available at: http://www.co3-project.eu/ wo3/wp-content/uploads/2011/12/CO3-D-2-6-Position-Paper-CO3-Tool-Support-Case-Studies-final20140718-2.pdf (accessed 4 October 2019).

Danneels, E. (2004), "Disruptive technology reconsidered: a critique and research agenda”, Journal of Product Innovation Management, Vol. 21 No. 4, pp. 246-258.

Durach, C.F., Kembro, J. and Wieland, A. (2017), "A new paradigm for systematic literature reviews in supply chain management”, Journal of Supply Chain Management, Vol. 53 No. 4, pp. 67-85.

Freeman, L.C. (1978), "Centrality in social networks conceptual clarification”, Social Networks, Vol. 1 No. 3, pp. 215-239.

Garfield, E. (1979), "Is citation analysis a legitimate evaluation tool?", Scientometrics, Vol. 1 No. 4, pp. 359-375.

Gazzard, N. and Montreuil, B. (2015), "A functional design for Physical Internet modular handling containers", 2nd International Physical Internet Conference, Mines ParisTech, July 6-July 8, Paris.

Glaser, B. and Strauss, A.L. (1967), The Discovery of Grounded Theory: Strategies for Qualitative Research, Aldine Publishing Company, Chicago, Illinois.

Gubbi, J., Buyya, R., Marusic, S. and Palaniswami, M. (2013), "Internet of Things (IoT): a vision, architectural elements, and future directions", Future Generation Computer Systems, Vol. 29 No. 7, pp. 1645-1660.

Hakimi, D., Montreuil, B. and Labarthe, O. (2009), "Supply web: concept and technology", 7th Annual International Symposium on Supply Chain Management, October 28-October 30, Toronto, Canada.

Hakimi, D., Montreuil, B. and Labarthe, O. (2010), "Supply web agent-based simulation platform", International Conference on Information Systems, Logistics and Supply Chain, April 14-April 16, Casablanca, Morocco.

Hakimi, D., Montreuil, B., Sarraj, R., Ballot, E. and Pan, S. (2012), "Simulating a Physical Internet enabled mobility web: the case of mass distribution in France", International Conference on Modeling, Optimization \& Simulation, June 6-June 8, Bordeaux, France, pp. 10-19.

Halldórsson, A., Kotzab, H., Mikkola, J.H. and Skjøtt-Larsen, T. (2007), "Complementary theories to supply chain management", Supply Chain Management: International Journal, Vol. 12 No. 4, pp. 284-296.

Hambleton, B. and Mannix, K. (2014), “The 3D supply network”, 1st International Physical Internet Conference, May 28-May 30, Université Laval, Quebec City, Canada.

Hambleton, B. and Mannix, K. (2015), "A quadruple case study for the physical internet: sunshine Nut and ES3", 2nd International Physical Internet Conference, Mines ParisTech, July 6-July 8, Paris, France.

Hao, G. and Gue, K.R. (2016), “A two-sided, high-density rail-rail hub”, 3rd International Physical Internet Conference, June 29-July 1, Georgia Institute of Technology, Atlanta, Georgia.

Hofman, W. (2016), "Evaluating five typologies on costs and requirements for hyperconnected logistics networks", 3rd International Physical Internet Conference, June 29-July 1, Georgia Institute of Technology, Atlanta, Georgia. 
Huschebeck, M. (2012), "Modulusha (modular logistics units in shared Co-modal networks). Cordis EU research", available at: http://www.modulushca.eu (accessed 25 August 2016).

Iacovou, C.L., Benbasat, I. and Dexter, A.S. (1995), "Electronic data interchange and small organizations: adoption and impact of technology", MIS Quarterly, Vol. 19 No. 4, pp. 465-485.

IPIC (2018), Mission, University of Groningen, available at: https://www.pi.events/about (accessed 21 July 2018).

Jacobs, K., Van Lent, C., Verstrepen, S. and Giventis, M.B. (2014), "Horizontal collaboration in fresh \& chilled retail distribution (Deliverable D4.5)", CO3 and TRI-VIZOR, available at: http://www.co3project.eu/wo3/wp-content/uploads/2011/12/CO3-Deliverable-Nestle-Pepsico-STEF-casestudy-1. pdf (accessed 12 August 2016).

Jacobs, K., Vercammen, S. and Verstrepen, S. (2013), "Creation of an orchestrated intermodal partnership between multiple shippers. CO3 Project and TRI-VIZOR", available at: http://www.co3-project.eu/ wo3/wp-content/uploads/2011/12/CO3-D-4-2-Test-case-multimodal-horizontal-collaboration.pdf (accessed 4 October 2019).

Ji, S.F., Peng, X.S. and Luo, R.J. (2019), “An integrated model for the production-inventory-distribution problem in the Physical Internet”, International Journal of Production Research, Vol. 57 No. 4, pp. 1000-1017.

Kache, F. and Seuring, S. (2017), "Challenges and opportunities of digital information at the intersection of big data analytics and supply chain management", International Journal of Operations and Production Management, Vol. 37 No. 1, pp. 10-36.

Kong, X.T., Chen, J., Luo, H. and Huang, G.Q. (2016), "Scheduling at an auction logistics centre with Physical Internet”, International Journal of Production Research, Vol. 54 No. 9, pp. 2670-2690.

Krogsgaard, A., Pisinger, D. and Thorsen, J. (2018), "A flow-first route-next heuristic for liner shipping network design”, Networks, Vol. 72 No. 3, pp. 358-381.

Landschützer, C., Ehrentraut, F. and Jodin, D. (2015), "Containers for the Physical Internet: requirements and engineering design related to FMCG logistics", Logistics Research, Vol. 8 No. 1, pp. 1-22.

Le Roch, Y., Ballot, E. and Perraudin, X. (2015), “A new framework for the management of returnable "containers" within open supply networks", Service Orientation in Holonic and Multi-Agent Manufacturing, November 5-November 6. University of Lorraine, Cambridge, UK.

Lin, I.C. and Cheng, C.Y. (2016), "Case study of Physical Internet for improving efficiency in solar cell industry", Journal of Ambient Intelligence and Humanized Computing, Vol. 9 No. 1, pp. 1-10.

Lounès, M. and Montreuil, B. (2011), "Towards a worldwide physical internet", TU International, Vol. 67, January, pp. 30-33, available at: https://www.picenter.gatech.edu/sites/default/files/ towards_a_worldwide_physical_internet2011.pdf (accessed 15 June 2016).

Markillie, P. (2006), The Physical Internet, The Economist, London, UK, available at: http://www. economist.com/node/7032165 (accessed 15 June 2016).

Mayring, P. (2014), Qualitative Content Analysis: Theoretical Foundation, Basic Procedures and Software Solution, self-published book, Klagenfurt, Austria.

Meller, R.D. and Ellis, K.P. (2012), Distances Reduce and Inventory Levels Change in a Physical Internet Network: A Result of the CELDi Physical Internet Project, Center for Excellence in Logistics and Distribution Fayetteville, Arkansas.

Meller, R.D., Ellis, K.P. and Loftis, B. (2012a), "From horizontal collaboration to the Physical Internet: quantifying the effects on sustainability and profits when shifting to interconnected logistics systems (Final research report)", available at: http:/faculty.ineg.uark.edu/rmeller/web/CELDiPI/index-PI.html (accessed 12 August 2016).

Meller, R.D., Montreuil, B., Thivierge, C. and Montreuil, Z. (2012b), "Functional design of Physical Internet facilities: a road-based transit center", 12th International Material Handling Research Colloquium, June 25-June 28, Gardanne, France.
PI as a new supply chain paradigm 
IJLM

31,2

Montreuil, B. (2010), "Toward a Physical Internet: a global sustainability focused collaborative networking initiative", PRO-VE 2010, St-Étienne, France, available at: http://www.uninova.pt/ prove09/2010/presentations/keynote-speaker2.pdf (accessed 26 June 2019).

Montreuil, B. (2011), "Towards a Physical Internet: meeting the global logistics sustainability grand challenge", Logistics Research, Vol. 3 Nos 2-3, pp. 71-87.

Montreuil, B. (2012), "Physical internet manifesto", CIRRELT Interuniversity Research Center on Enterprise Networks, Logistics and Transportation, available at: http://physicalinternetinitiative. org/Physical\%20Internet\%20Manifesto\%20Version\%201.11\%202012-11-20.pdf (accessed 25 April 2016).

Montreuil, B., Ballot, E. and Fontane, F. (2012), “An open logistics interconnection model for the Physical Internet”, 14th IFAC Symposium on Information Control Problems in Manufacturing, May 23-May 25, Bucharest, Romania, pp. 327-332.

Montreuil, B., Ballot, E. and Tremblay, W. (2014), "Modular design of Physical Internet transport, handling and packaging containers", in Smith, J., Ellis, K., De Koster, R., Lavender, S., Montreuil, B. and Ogle, M. (Eds), Progress in Material Handling Research, MHI, Charlotte, North Carolina, pp. 1-13.

Montreuil, B., Meller, R.D. and Ballot, E. (2010), “Towards a Physical Internet: the impact on logistics facilities and material handling systems design and innovation”, 11th International Material Handling Research Colloquium, 21 June-24 June, Milwaukee, Wisconsin.

Montreuil, B., Meller, R.D. and Ballot, E. (2013a), "Physical internet foundations", CIRRELT, Université Laval, Québec, Canada, available at: http://www.fsa.ulaval.ca/sirul/2012-015.pdf (accessed 25 April 2016).

Montreuil, B., Meller, R.D., Thivierge, C. and Montreuil, Z. (2013b), "Functional design of Physical Internet facilities: a unimodal road-based crossdocking hub", CIRRELT, Université Laval, Québec, Canada, available at: https://www.cirrelt.ca/DocumentsTravail/CIRRELT-FSA-2013-15. pdf (accessed 25 April 2016).

Naccache, S., Montreuil, B., Sohrabi, H., Barriault, F. and Brotherton, E. (2014), "From integrated to interconnected B2C e-commerce distribution: an agent-based simulation assessment", 1st International Physical Internet Conference, May 28-May 30, Université Laval, Quebec City.

Oktaei, P., Lehoux, N. and Montreuil, B. (2014), "Designing business models for Physical Internet transit centers", 1st International Physical Internet Conference, May 28-May 30, Université Laval, Quebec City.

Olhager, J., Pashaei, S. and Sternberg, H. (2015), "Design of global production and distribution networks: a literature review and research agenda", International Journal of Physical Distribution and Logistics Management, Vol. 45 Nos 1/2, pp. 138-158.

Othmane, I.B., Rekik, M. and Mellouli, S. (2014), "Impact of shipper collaboration on carriers selection in reputation-based transportation auctions", 1st International Physical Internet Conference, May 28-May 30, Université Laval, Quebec City.

Pach, C., Berger, T., Adam, E., Bonte, T. and Sallez, Y. (2014), "Proposition of a potential fields approach to solve routing in a rail-road PI-hub", 1st International Physical Internet Conference, May 28-May 30, Université Laval, Quebec City.

Pan, S., Ballot, E., Huang, G.Q. and Montreuil, B. (2017), "Physical Internet and interconnected logistics services: research and applications", International Journal of Production Research, Vol. 55 No. 9, pp. 2603-2609.

Pan, S., Nigrelli, M., Ballot, E. and Sarraj, R. (2013), "Performance assessment of distributed inventory in Physical Internet", 43rd International Conference on Computers and Industrial Engineering, October 16-October 18, Hong Kong, China.

Pan, S., Chen, C. and Zhong, R.Y. (2015a), "Using taxi fleet to collect e-commerce reverse flows in city: a simulation study", 2nd International Physical Internet Conference, July 6-July 8, Mines ParisTech, Paris. 
Pan, S., Nigrelli, M., Ballot, E., Sarraj, R. and Yang, Y. (2015b), "Perspectives of inventory control models in the Physical Internet: a simulation study", Computers and Industrial Engineering, Vol. 84 June, pp. 122-132.

Puskás, E. and Bohács, G. (2019), "Physical Internet: a novel application area for Industry 4.0", International Journal of Engineering and Management Sciences, Vol. 4 No. 1, pp. 152-161.

Qiao, B., Pan, S. and Ballot, E. (2016a), "Dynamic pricing model for less-than-truckload carriers in the Physical Internet”, Journal of Intelligent Manufacturing, Vol. 30 No 7, pp. 2631-2643.

Qiao, B., Pan, S. and Ballot, E. (2016b), "Less-than-truckload dynamic pricing model in Physical Internet", 5th Institute of Industrial Engineers Asian Conference, July 21-July 22, Hong Kong, China.

Rousseau, D., Manning, J. and Denyer, D. (2008), "Evidence in management and organizational science: assembling the field's full weight of scientific knowledge through syntheses", The Academy of Management Annals, Vol. 2 No. 1, pp. 475-515.

Sallez, Y., Berger, T., Bonte, T. and Trentesaux, D. (2015a), "Proposition of a hybrid control architecture for the routing in a Physical Internet cross-docking hub", 15th IFAC Symposium on Information Control Problems in Manufacturing, May 11-May 13, Ottawa, Canada, pp. 1978-1983.

Sallez, Y., Montreuil, B. and Ballot, E. (2015b), "On the activeness of Physical Internet containers", Service Orientation in Holonic and Multi-Agent Manufacturing, Springer, Basel, Switzerland, pp. 259-269.

Sallez, Y., Pan, S., Montreuil, B., Berger, T. and Ballot, E. (2016), "On the activeness of intelligent Physical Internet containers", Computers in Industry, Vol. 81 September, pp. 96-104.

Sarraj, R., Ballot, E., Pan, S., Hakimi, D. and Montreuil, B. (2014a), "Interconnected logistic networks and protocols: simulation-based efficiency assessment", International Journal of Production Research, Vol. 52 No. 11, pp. 3185-3208.

Sarraj, R., Ballot, E., Pan, S. and Montreuil, B. (2014b), "Analogies between Internet network and logistics service networks: challenges involved in the interconnection", Journal of Intelligent Manufacturing, Vol. 25 No. 6, pp. 1207-1219.

Schoen, Q., Fontanili, F., Lauras, M., Truptil, S. and Anquetil, A.G. (2016), "PI-based automated diagnosis: the blood supply chain perspective", 3rd International Physical Internet Conference, June 29-July 1, Georgia Institute of Technology Atlanta, GA.

Schroth, C. and Janner, T. (2007), "Web 2.0 and SOA: converging concepts enabling the Internet of services”, IT Professional, Vol. 9 No. 3, pp. 36-41.

Sohrabi, H. and Montreuil, B. (2011), "From private supply networks and shared supply webs to Physical Internet enabled open supply webs", Adaptation and Value Creating Collaborative Networks, Springer, Basel, Switzerland, pp. 235-244.

Sternberg, H. and Norrman, A. (2017), “The Physical Internet: review, analysis and future research agenda”, International Journal of Physical Distribution and Logistics Management, Vol. 47 No. 8, pp. 736-762.

Tran-Dang, H., Krommenacker, N. and Charpentier, P. (2015), "Enhancing the functionality of Physical Internet containers by wireless sensor networks", 2nd International Physical Internet Conference, July 6-July 8, Mines ParisTech, Paris.

Tran-Dang, H., Krommenacker, N. and Charpentier, P. (2016), "Containers monitoring through the Physical Internet: a spatial 3D model based on wireless sensor networks", International Journal of Production Research, Vol. 55 No. 9, pp. 2650-2663.

Tranfield, D., Denyer, D. and Smart, P. (2003), "Towards a methodology for developing evidenceinformed management knowledge by means of systematic review", British Journal of Management, Vol. 14 No. 3, pp. 207-222.

Treiblmaier, H. (2018), "The impact of the blockchain on the supply chain: a theory-based research framework and a call for action”, Supply Chain Management: International Journal, Vol. 23 No. 6, pp. 545-559.

PI as a new supply chain paradigm 
IJLM

31,2

Treiblmaier, H. (2019), "Combining Blockchain technology and the Physical Internet to achieve triple bottom line sustainability: a comprehensive research agenda for modern logistics and supply chain management", Logistics, Vol. 3 No. 10, pp. 1-13.

Treiblmaier, H., Mirkovski, K. and Lowry, P.B. (2016), "Conceptualizing the Physical Internet: literature review, implications and directions for future research", 11th CSCMP Annual European Research Seminar, May 12-May 13, Vienna, Austria.

Tretola, G. and Verdino, V. (2014), "A collaborative approach for managing data and processes in the Physical Internet using modular logistics", 1st International Physical Internet Conference, May 28-May 30, Université Laval, Quebec City.

Tretola, G. and Verdino, V. (2015), "High level ICT architecture for modular logistics", 2nd International Physical Internet Conference, July 6-July 8, Mines ParisTech, Paris.

Tretola, G., Verdino, V. and Biggi, D. (2015), “A common data model for the Physical Internet”, 2nd International Physical Internet Conference, July 6-July 8, Mines ParisTech, Paris.

Venkatadri, U., Krishna, K.S. and Ülkü, M.A. (2016), "On Physical Internet logistics: modeling the impact of consolidation on transportation and inventory costs", IEEE Transactions on Automation Science and Engineering, Vol. 13 No. 4, pp. 1517-1527.

Verstrepen, S. and Jacobs, K. (2012), "Creation of an orchestrated horizontal collaboration for road bundling between two shippers", TriVizor and CO3-project, available at: http://www.co3project.eu/wo3/wp-content/uploads/2011/12/CO ${ }^{3}$-D4.1Test_Case_road_bundling_JSP_Hammer werk-Executive-Summary.pdf (accessed 12 August 2016).

Wang, J.Q., Fan, G.Q., Yan, F.Y., Zhang, Y.F. and Sun, S.D. (2016), "Research on initiative scheduling mode for a Physical Internet-based manufacturing system", International Journal of Advanced Manufacturing Technology, Vol. 84 Nos 1-4, pp. 47-58.

Watson, R.T. (2015), "Beyond being systematic in literature reviews in IS", Journal of Information Technology, Vol. 30 No. 2, pp. 185-187.

Wilding, R., Wagner, B., Colicchia, C. and Strozzi, F. (2012), "Supply chain risk management: a new methodology for a systematic literature review", Supply Chain Management: International Journal, Vol. 17 No. 4, pp. 403-418.

Xu, X., Pan, S. and Ballot, E. (2013), “A sharing mechanism for superadditive and non-superadditive logistics cooperation", 5th International Conference on Industrial Engineering and Systems Management, October 28-October 30, IEEE, Agdal.

Yang, Y., Pan, S. and Ballot, E. (2015a), "An inventory control model with interconnected logistic services for vendor inventory management", 2nd International Physical Internet Conference, July 6-July 8, Mines ParisTech, Paris.

Yang, Y., Pan, S. and Ballot, E. (2015b), "A model to take advantage of Physical Internet for vendor inventory management", 15th IFAC Symposium on Information Control Problems in Manufacturing, May 13-May 15, Ottawa, Canada, pp. 1990-1995.

Yang, Y., Pan, S. and Ballot, E. (2016), "Mitigating supply chain disruptions through interconnected logistics services in the Physical Internet”, International Journal of Production Research, Vol. 55 No. 14, pp. 3970-3983.

Zhang, Y., Liu, S., Liu, Y. and Li, R. (2016), "Smart box-enabled product-service system for cloud logistics", International Journal of Production Research, Vol. 54 No. 22, pp. 6693-6706.

Zhong, R.Y., Gong, H., Xu, C. and Lu, S. (2016), "Physical Internet-enabled manufacturing execution system for intelligent workshop production", International Journal of Signal Processing, Image Processing, and Pattern Recognition, Vol. 9 No. 6, pp. 121-132.

Zhong, R.Y., Peng, Y., Fang, J., Xu, G., Xue, F., Zou, W. and Huang, G.Q. (2015), “Towards Physical Internet-enabled prefabricated housing construction in Hong Kong", 15th IFAC Symposium on Information Control Problems in Manufacturing, May 13-May 15, Ottawa, Canada, pp. 1079-1086. 


\section{References in Appendix 1}

Ballot, E., Montreuil, B. and Thivierge, C. (2012), "Functional design of Physical Internet facilities: a roadrail hub”, in Montreuil, B., Carrano, A., De Kostner, M.M.R., Gue, K.R., Ogle, M. and Smith, J. (Eds), Progress in Material Handling Research, Material Handling Industry of America, Charlotte, North California.

Biermasz, J. and Louws, M. (2014), "Legal framework transformation”, CO3 Project, available at: http:// www.co3-project.eu/wo3/wp-content/uploads/2011/12/CO3-D-2-9-Legal-Framework-excl-contr.august-2014.pdf (accessed 12 August 2016).

Chen, C., Pan, S., Wang, Z. and Zhong, R.Y. (2017), "Using taxis to collect citywide e-commerce reverse flows: a crowdsourcing solution”, International Journal of Production Research, Vol. 55 No. 7, pp. 1833-1844.

Crainic, T.G. and Montreuil, B. (2015), "Physical Internet enabled interconnected city logistics”, Centre Interuniversitaire de Recherche sur les Réseaux d'Entreprise, la Logistique et le Transport (CIRRELT), available at: https:/www.cirrelt.ca/DocumentsTravail/CIRRELT-2015-13.pdf (accessed 19 May 2017).

Cruijssen, F. (2012a), "Framework for collaboration (deliverable D2.1)", CO3 Project, available at: http:// www.co3-project.eu/wo3/wp-content/uploads/2011/12/CO3-D-2-1-Framework-for-collaboration-fullreport-2.pdf (accessed 12 August 2016).

Eye for Transport (2011), "North American horizontal collaboration in the supply chain", available at: http://faculty.ineg.uark.edu/rmeller/web/CELDi-PI/NAHCSC-2011.pdf (accessed 22 August 2016).

Fazili, M., Venkatadri, U., Cyrus, P. and Tajbakhsh, M. (2017), "Physical Internet, conventional and hybrid logistic systems: a routing optimisation-based comparison using the Eastern Canada road network case study”, International Journal of Production Research, Vol. 55 No. 9, pp. 2703-2730.

Franklin, R. and Spinler, S. (2011), "Shared warehouses: sharing risks and increasing eco-efficiency", International Commerce Review, Vol. 10 No. 1, pp. 22-31.

Furtado, P. and Frayret, J.M. (2014), "Impact of resource sharing of freight transportation", 1st International Physical Internet Conference, May 28 - May 30, Université Laval, Quebec City.

Genta, S. and Cruijssen, F. (2013), "Web accessible set of methods and tools supporting collaboration and co-modality (Deliverable D2.6)”, CO3 Project, available at: http://www.elupeg.com/ downloads/2388\%5E (accessed 12 August 2016).

Hakimi, D., Montreuil, B., Sarraj, R., Ballot, E. and Pan, S. (2012a), "Simulating a Physical Internet enabled mobility web: the case of mass distribution in France", International Conference on Modeling, Optimization \& Simulation, June 6-June 8, Bordeaux, France, pp. 10-19.

Hofman, W. (2015), "Federated platforms for seamless interoperability in the physical Internet", 2nd International Physical Internet Conference, July 6-July 8, Mines ParisTech, Paris.

Jacobs, K., Van Lent, C., Verstrepen, S. and Giventis, M.B. (2014), "Horizontal collaboration in fresh \& chilled retail distribution (Deliverable D4.5)", CO3 and TRI-VIZOR, available at: http://www.co3project.eu/wo3/wp-content/uploads/2011/12/CO3-Deliverable-Nestlé-Pepsico-STEF-case-study1.pdf (accessed 12 August 2016).

Kitchenham, B. and Brereton, P. (2013), “A systematic review of systematic review process research in software engineering”, Information and Software Technology, Vol. 55 No. 12, pp. 2049-2075.

Kong, X.T., Chen, J., Luo, H. and Huang, G.Q. (2016), "Scheduling at an auction logistics centre with Physical Internet”, International Journal of Production Research, Vol. 54 No. 9, pp. 2670-2690.

Koulougli, K., Chaabane, A. and Amodeo, L. (2015), “Assessment of transportation collaboration in global logistics”, 2nd International Physical Internet Conference, July 6 - July 8, Mines ParisTech, Paris.

Landschützer, C., Ehrentraut, F. and Jodin, D. (2015), "Containers for the Physical Internet: requirements and engineering design related to FMCG logistics”, Logistics Research, Vol. 8 No. 1, pp. 1-22.

PI as a new supply chain paradigm 
Lin, I.C. and Cheng, C.Y. (2016), "Case study of Physical Internet for improving efficiency in solar cell industry", Journal of Ambient Intelligence and Humanized Computing, Vol. 9 No. 1, pp. 1-10.

Lin, Y.H., Meller, R.D., Ellis, K.P., Thomas, L.M. and Lombardi, B.J. (2014), "A decomposition-based approach for the selection of standardized modular containers", International Journal of Production Research, Vol. 52 No. 15, pp. 4660-4672.

Maslarić, M., Nikoličić, S. and Mirčetić, D. (2016), "Logistics response to the Industry 4.0: the physical internet”, Open Engineering, Vol. 6 No. 1, pp. 511-517.

Meller, R.D. and Ellis, K.P. (2012a), Standardizing container sizes saves space in the trailer: A result of the CELDi physical Internet project, Center for Excellence in Logistics and Distribution, Fayetteville, AR, available at: http://faculty.ineg.uark.edu/rmeller/web/CELDi-PI/Results\% 20Summary.pdf (accessed 05 February 2016).

Meller, R.D., Montreuil, B., Thivierge, C. and Montreuil, Z. (2012c), "Functional design of Physical Internet facilities: a road-based transit center", 12th International Material Handling Research Colloquium, June 25-June 28, Gardanne, France.

Mohamed, I.B., Klibi, W., Labarthe, O., Deschamps, J.-C. and Babai, Z.M. (2017), "Modelling and solution approaches for the interconnected city logistics", International Journal of Production Research, Vol. 55 No. 9, pp. 2664-2684.

Montreuil, B. (2010), "Toward a Physical Internet: a global sustainability focused collaborative networking initiative", PRO-VE 2010, St-Étienne, France, available at: http:/www.uninova.pt/ prove09/2010/presentations/keynote-speaker2.pdf (accessed 26 June 2019).

Montreuil, B. (2012), "Physical internet manifesto", CIRRELT Interuniversity Research Center on Enterprise Networks, Logistics and Transportation, available at: http://physicalinternetinitiative. org/Physical\%20Internet \%20Manifesto\%20Version\%201.11\%202012-11-20.pdf (accessed 25 April 2016).

Montreuil, B., Meller, R.D. and Ballot, E. (2013), "Physical internet foundations", CIRRELT, Université Laval, Québec, Canada, available at: http://www.fsa.ulaval.ca/sirul/2012-015.pdf (accessed 25 April 2016).

Oktaei, P., Hakimi, D., Lehoux, N., Montreuil, B. and Cloutier, C. (2015), "Impact of geographic locations on the business model of Physical Internet enabled transit centers", 2nd International Physical Internet Conference, July 6-July 8, Mines ParisTech, Paris.

Oktaei, P., Lehoux, N. and Montreuil, B. (2014), "Designing business models for Physical Internet transit centers", 1st International Physical Internet Conference, May 28-May 30, Université Laval, Quebec City.

Pach, C., Berger, T., Adam, E., Bonte, T. and Sallez, Y. (2014), "Proposition of a potential fields approach to solve routing in a rail-road PI-hub", 1st International Physical Internet Conference, May 28-May 30, Université Laval, Quebec City.

Pan, S., Nigrelli, M., Ballot, E., Sarraj, R. and Yang, Y. (2015), "Perspectives of inventory control models in the Physical Internet: a simulation study", Computers and Industrial Engineering, Vol. 84 June, pp. 122-132.

Qiao, B., Pan, S. and Ballot, E. (2016), "Less-than-truckload dynamic pricing model in Physical Internet", 5th Institute of Industrial Engineers Asian Conference, July 21-July 22, Hong Kong, China.

Rossi, S. (2012), "Challenges for co-modality in a collaborative environment", CO3 Project, available at: http://www.co3-project.eu/wo3/wp-content/uploads/2011/12/CO \%C2\%B3-D2-3Co-Modality-and-Collaboration-Executive-Summary_def1.pdf (accessed 12 August 2016).

Rougès, J.F. and Montreuil, B. (2014), "Crowdsourcing delivery: New interconnected business models to reinvent delivery", 1st International Physical Internet Conference, May 28 - May 30, Université Laval, Quebec City.

Sallez, Y., Berger, T., Bonte, T. and Trentesaux, D. (2015a), "Proposition of a hybrid control architecture for the routing in a Physical Internet cross-docking hub", 15th IFAC Symposium 
on Information Control Problems in Manufacturing, May 11-May 13, Ottawa, Canada, pp. 1978-1983.

Sallez, Y., Montreuil, B. and Ballot, E. (2015b), "On the activeness of Physical Internet containers", Service Orientation in Holonic and Multi-Agent Manufacturing, Springer, Basel, Switzerland, pp. 259-269.

Sallez, Y., Pan, S., Montreuil, B., Berger, T. and Ballot, E. (2016), "On the activeness of intelligent Physical Internet containers", Computers in Industry, Vol. 81 September, pp. 96-104.

Sarraj, R., Ballot, E., Pan, S., Hakimi, D. and Montreuil, B. (2014a), "Interconnected logistic networks and protocols: simulation-based efficiency assessment", International Journal of Production Research, Vol. 52 No. 11, pp. 3185-3208.

Sarraj, R., Ballot, E., Pan, S. and Montreuil, B. (2014b), "Analogies between Internet network and logistics service networks: challenges involved in the interconnection", Journal of Intelligent Manufacturing, Vol. 25 No. 6, pp. 1207-1219.

Sohrabi, H. and Montreuil, B. (2011), "From private supply networks and shared supply webs to Physical Internet enabled open supply webs", Adaptation and Value Creating Collaborative Networks, Springer, Basel, Switzerland, pp. 235-244.

Sohrabi, H., Montreuil, B. and Klibi, W. (2015), "An optimization-based investigation of exploiting Physical Internet-enabled interconnected distribution system”, 2nd International Physical Internet Conference, July 6 - July 8, Mines ParisTech, Paris.

Sternberg, H. and Norrman, A. (2017), "The Physical Internet: review, analysis and future research agenda”, International Journal of Physical Distribution and Logistics Management, Vol. 47 No. 8, pp. 736-762.

Tran-Dang, H., Krommenacker, N. and Charpentier, P. (2015), "Enhancing the functionality of Physical Internet containers by wireless sensor networks", 2nd International Physical Internet Conference, July 6-July 8, Mines ParisTech, Paris.

Tran-Dang, H., Krommenacker, N. and Charpentier, P. (2016), "Containers monitoring through the Physical Internet: a spatial 3D model based on wireless sensor networks", International Journal of Production Research, Vol. 55 No. 9, pp. 2650-2663.

Tretola, G. and Verdino, V. (2014), "A collaborative approach for managing data and processes in the Physical Internet using modular logistics", 1st International Physical Internet Conference, May 28-May 30, Université Laval, Quebec City.

Tretola, G. and Verdino, V. (2015), "High level ICT architecture for modular logistics", 2nd International Physical Internet Conference, July 6-July 8, Mines ParisTech, Paris.

Venkatadri, U., Krishna, K.S. and Ülkü, M.A. (2016), "On Physical Internet logistics: modeling the impact of consolidation on transportation and inventory costs", IEEE Transactions on Automation Science and Engineering, Vol. 13 No. 4, pp. 1517-1527.

Verstrepen, S. and Van Den Bossche, L. (2013), "Retail inbound horizontal collaboration: Case study in Belgium”, available at: http://www.co3-project.eu/wo3/wp-content/uploads/2011/12/spar_retail_ bundling_of_loads.pdf (accessed 10 August 2016).

Walha, F., Bekrar, A., Chaabane, S. and Loukil, T. (2014), "A rail-road PI-hub allocation problems: Model and heuristic", 1st International Physical Internet Conference, May 28 - May 30, Université Laval, Quebec City.

Walha, F., Chaabane, S., Bekrar, A. and Loukil, T. M. (2015), "A simulated annealing metaheuristic for a rail-road PI-hub allocation problem", in Borangiu, T, Thomas, A, Trentesaux, D. (Eds), Service Orientation in Holonic and Multi-Agent Manufacturing, Springer, Basel, Switzerland, pp. 307-314.

Wang, J.Q., Fan, G.Q., Yan, F.Y., Zhang, Y.F. and Sun, S.D. (2016), "Research on initiative scheduling mode for a Physical Internet-based manufacturing system", International Journal of Advanced Manufacturing Technology, Vol. 84 Nos 1-4, pp. 47-58.

PI as a new supply chain paradigm 
Xu, X., Pan, S. and Ballot, E. (2013), “A sharing mechanism for superadditive and non-superadditive logistics cooperation", 5th International Conference on Industrial Engineering and Systems Management, October 28-October 30, IEEE, Agdal.

Yang, Y., Pan, S. and Ballot, E. (2015), "A model to take advantage of Physical Internet for vendor inventory management", 15th IFAC Symposium on Information Control Problems in Manufacturing, May 13-May 15, Ottawa, Canada, pp. 1990-1995.

Yang, Y., Pan, S. and Ballot, E. (2016), "Mitigating supply chain disruptions through interconnected logistics services in the Physical Internet", International Journal of Production Research, Vol. 55 No. 14, pp. 3970-3983.

Yang, Y., Pan, S. and Ballot, E. (2017), "Innovative vendor-managed inventory strategy exploiting interconnected logistics services in the Physical Internet", International Journal of Production Research, Vol. 55 No. 9, pp. 2685-2702.

Yao, J. (2016). "Optimisation of one-stop delivery scheduling in online shopping based on the physical internet", International Journal of Production Research, Vol. 55 No. 2, pp. 1-19.

Zhang, Y., Liu, S., Liu, Y. and Li, R. (2016), "Smart box-enabled product-service system for cloud logistics", International Journal of Production Research, Vol. 54 No. 22, pp. 6693-6706.

Zhong, R.Y., Peng, Y., Fang, J., Xu, G., Xue, F., Zou, W. and Huang, G.Q. (2015), “Towards Physical Internet-enabled prefabricated housing construction in Hong Kong", 15th IFAC Symposium on Information Control Problems in Manufacturing, May 13-May 15, Ottawa, Canada, pp. 1079-1086.

Zhong, R.Y., Xu, C., Chen, C. and Huang, G.Q. (2017), "Big data analytics for Physical Internet-based intelligent manufacturing shop floors”, International Journal of Production Research, Vol. 55 No. 9, pp. 2610-2621. 
Appendix 1
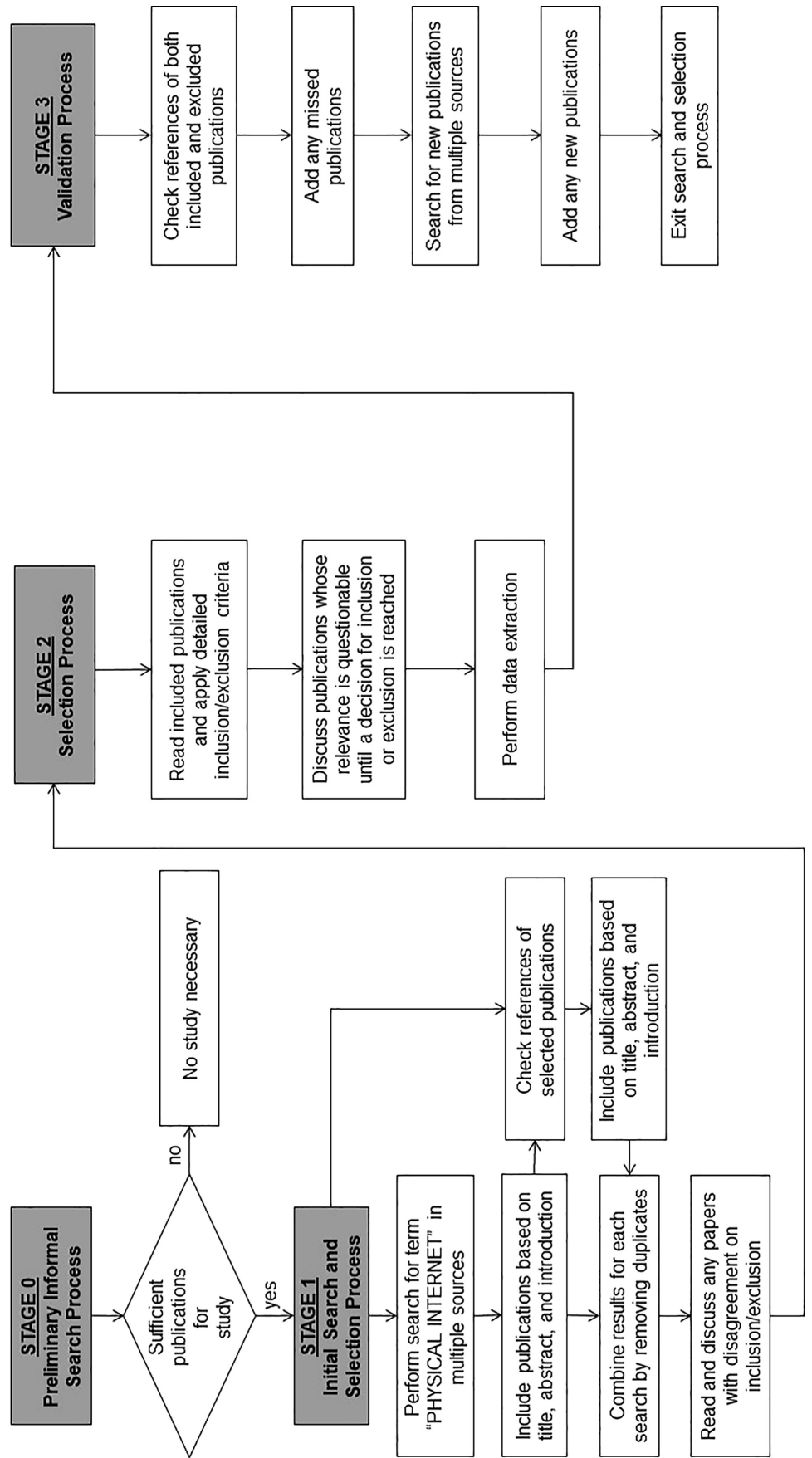

PI as a new

supply chain paradigm

277
Figure A1.

Stages of the search, selection and validation processes [cf. (Kitchenham and Brereton, 2013)] 
IJLM

31,2

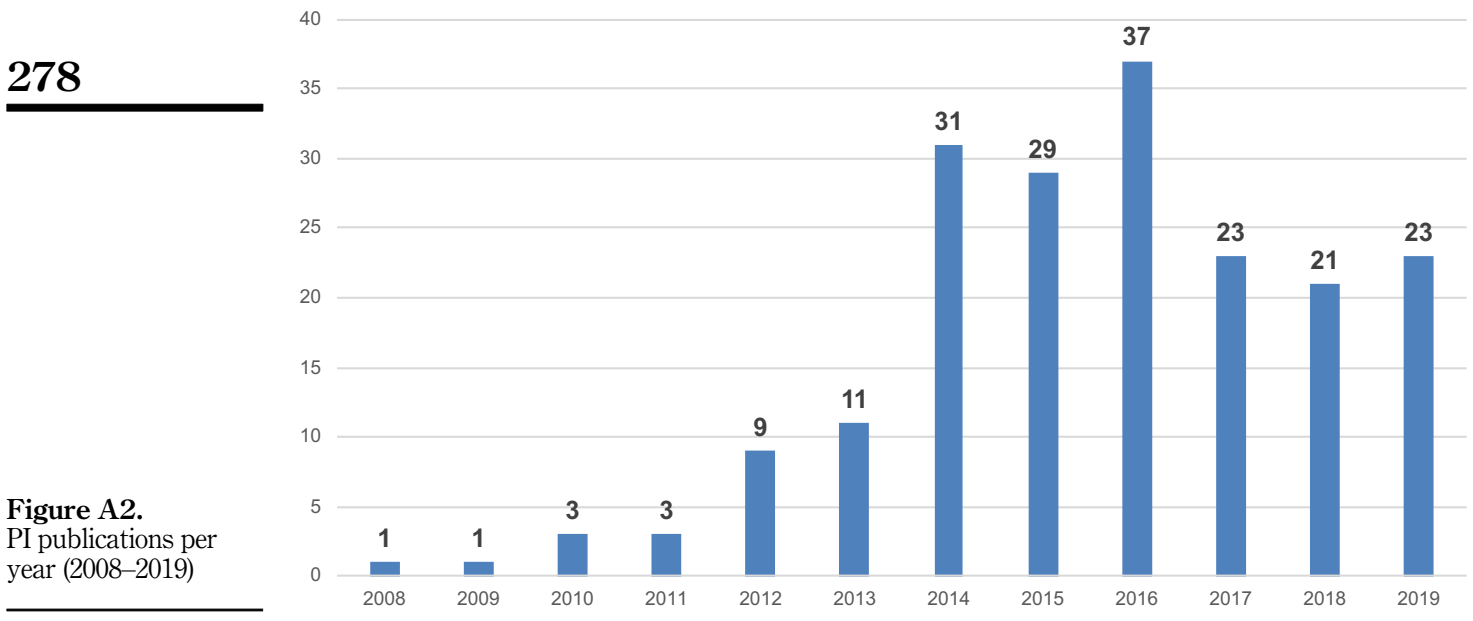


PI as a new

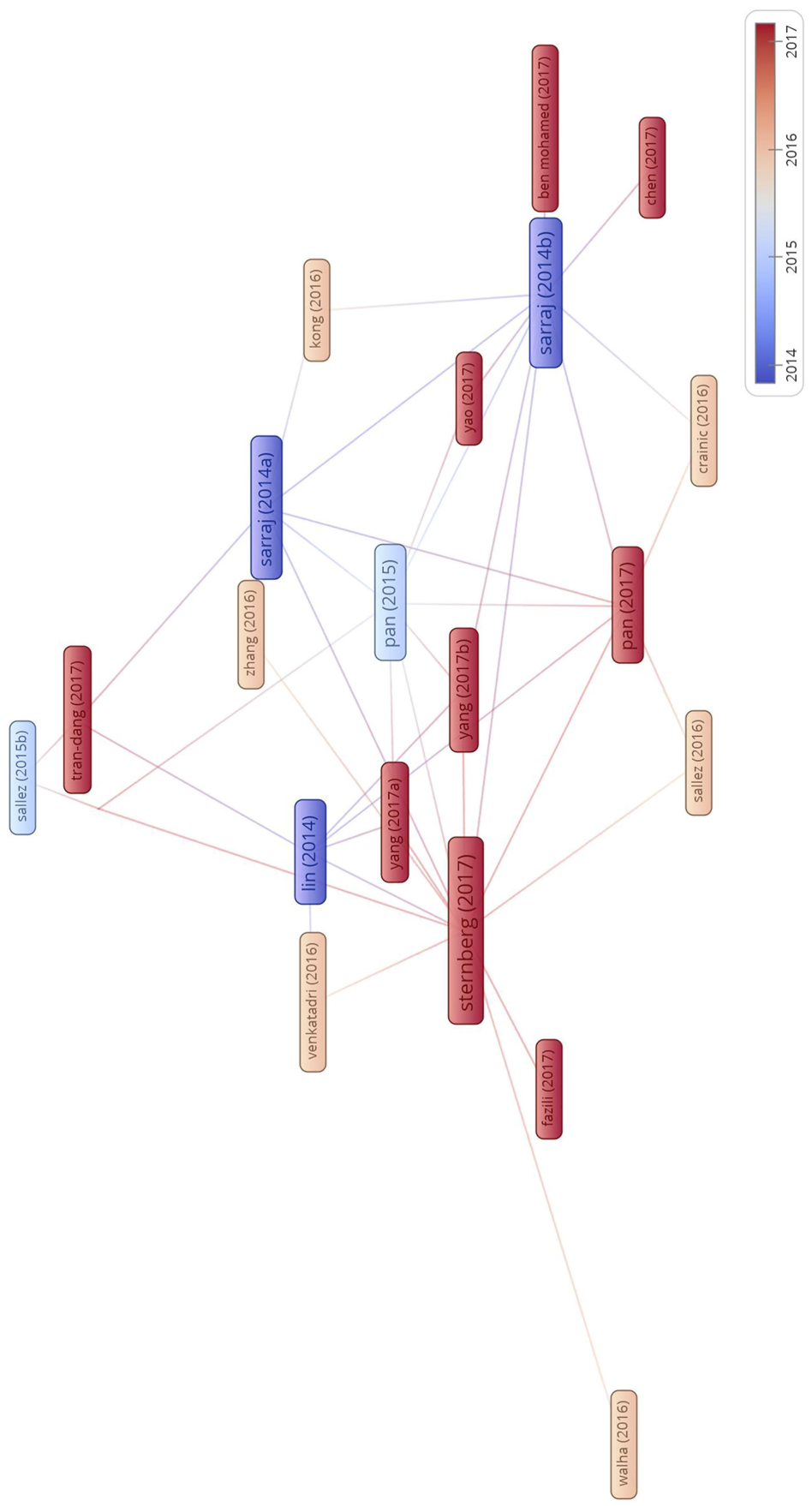

supply chain

paradigm

279

Figure A3.

Citation network of the 22 cited PI publications 
IJLM
31,2

280

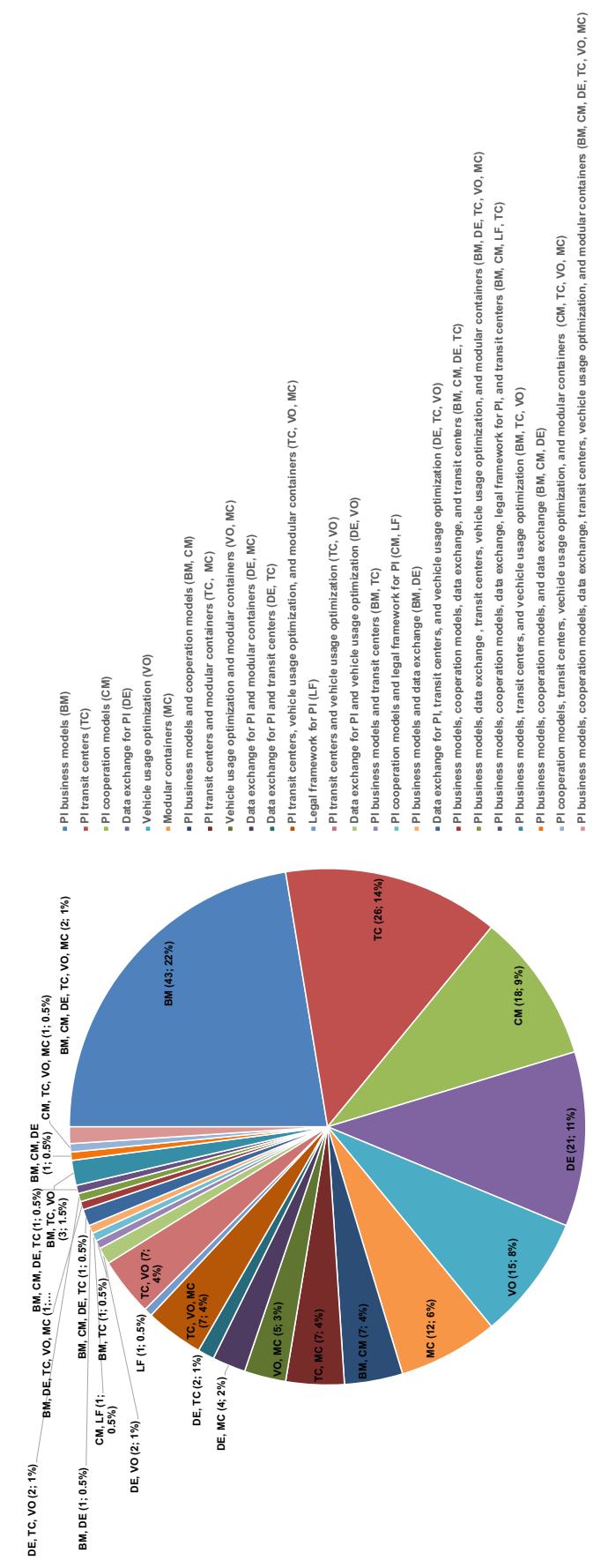

Figure A4.

PI themes in percentages 
PI as a new

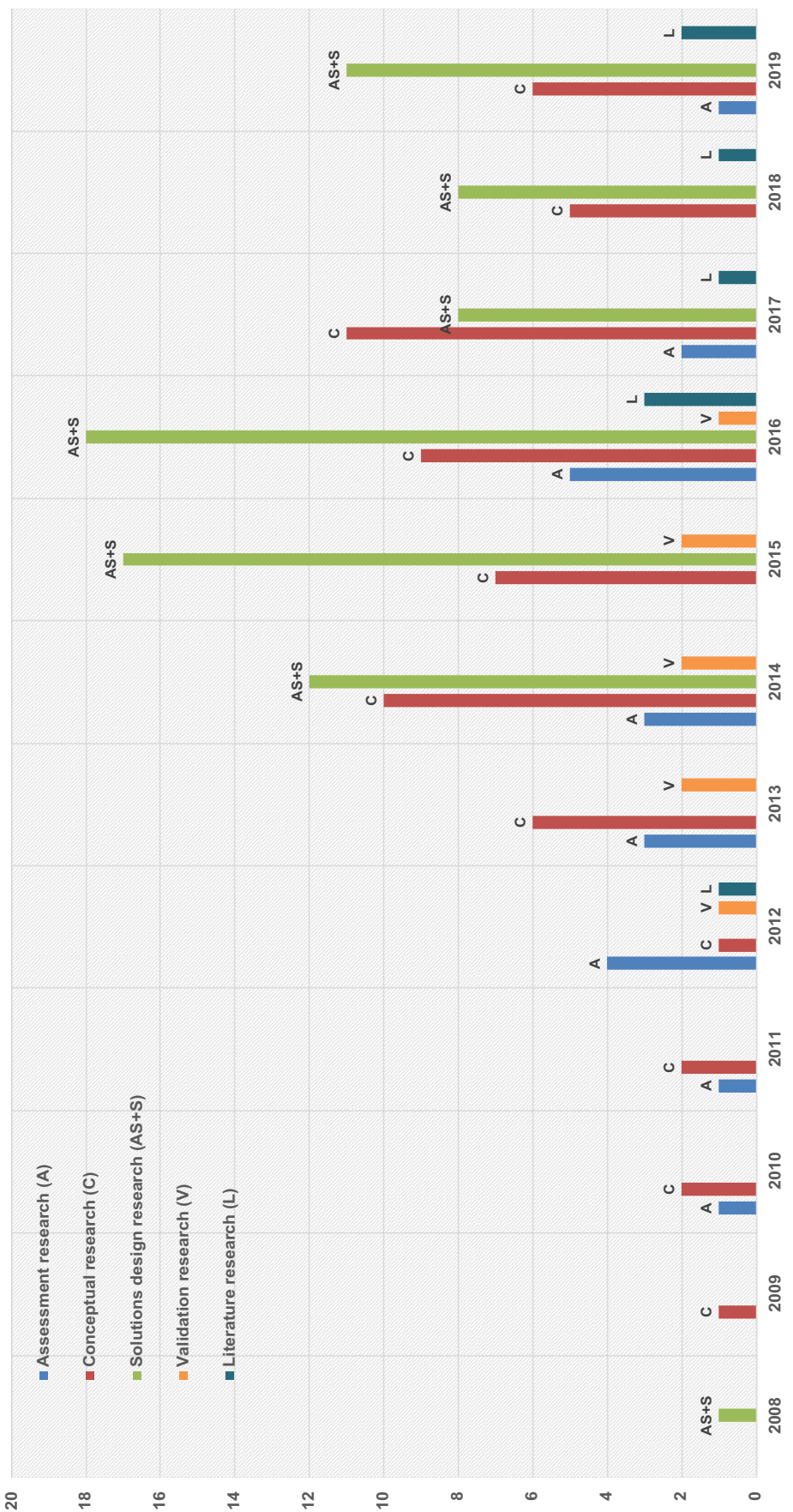

supply chain

paradigm

281

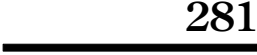




\begin{tabular}{l} 
IJLM \\
31,2 \\
\\
$\mathbf{2 8 2}$ \\
\hline
\end{tabular}

Table AI.

Cited publications in the PI literature*

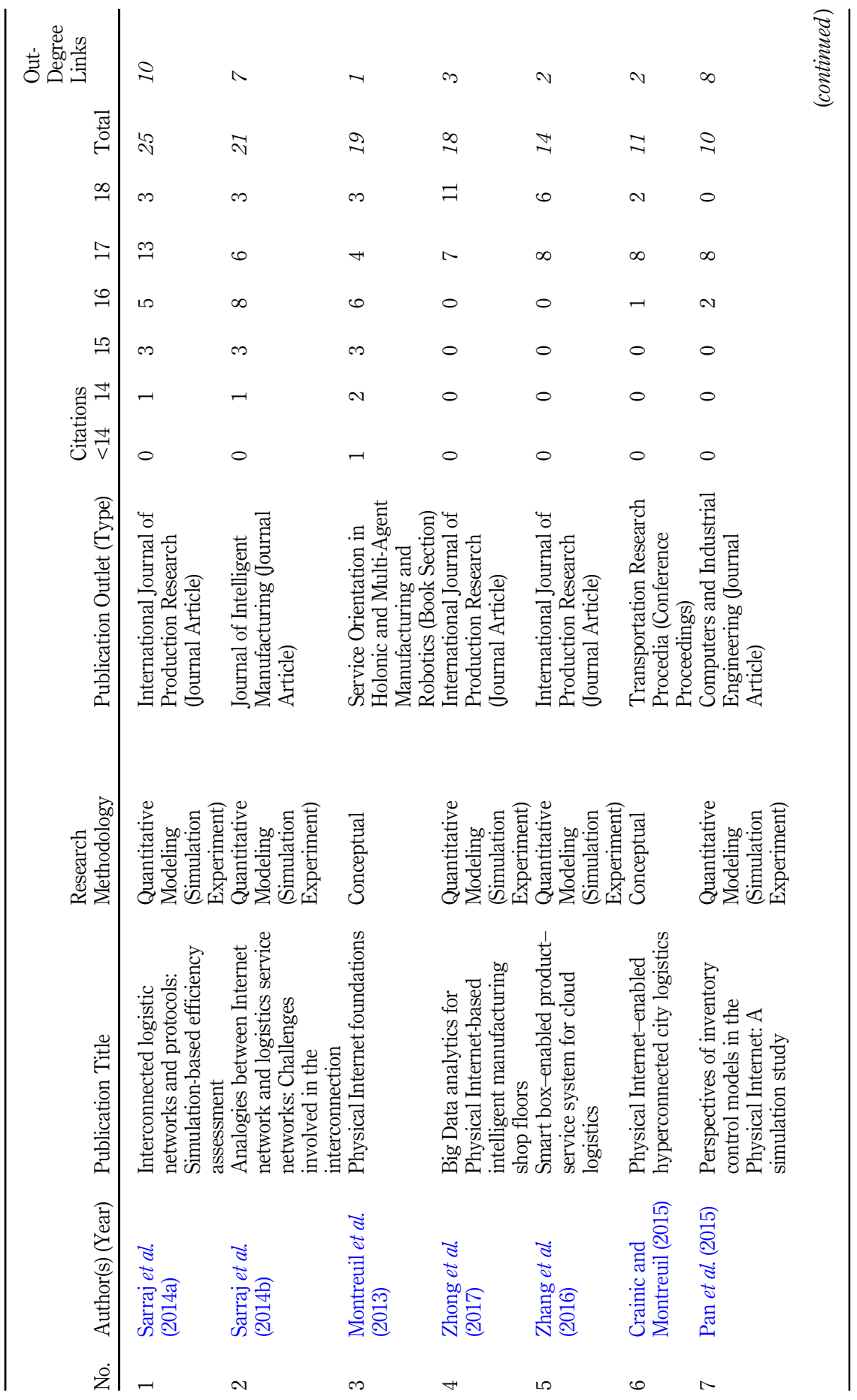




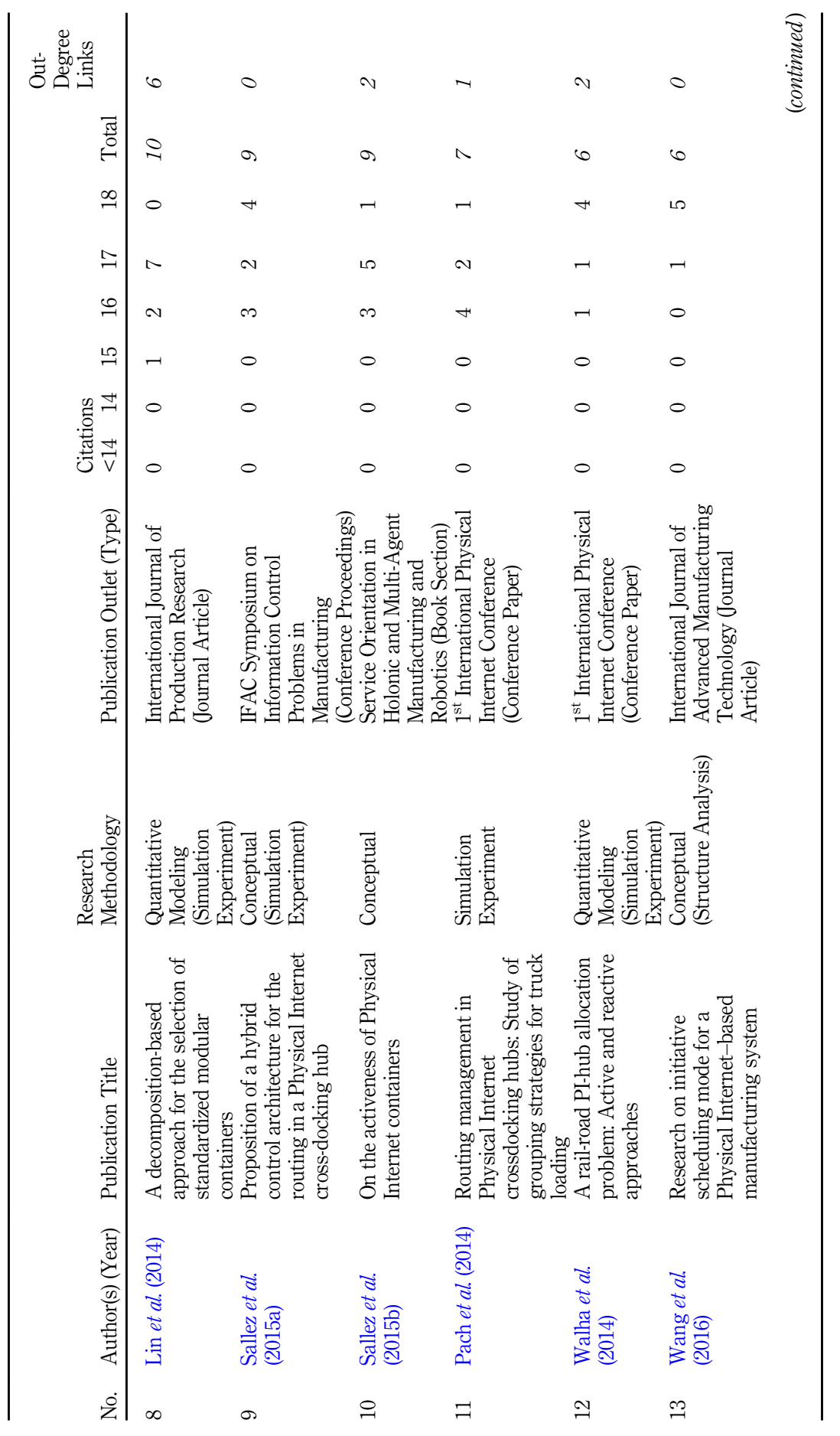

PI as a new supply chain paradigm

283

Table AI. 


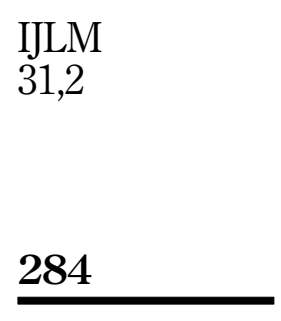

Table AI.

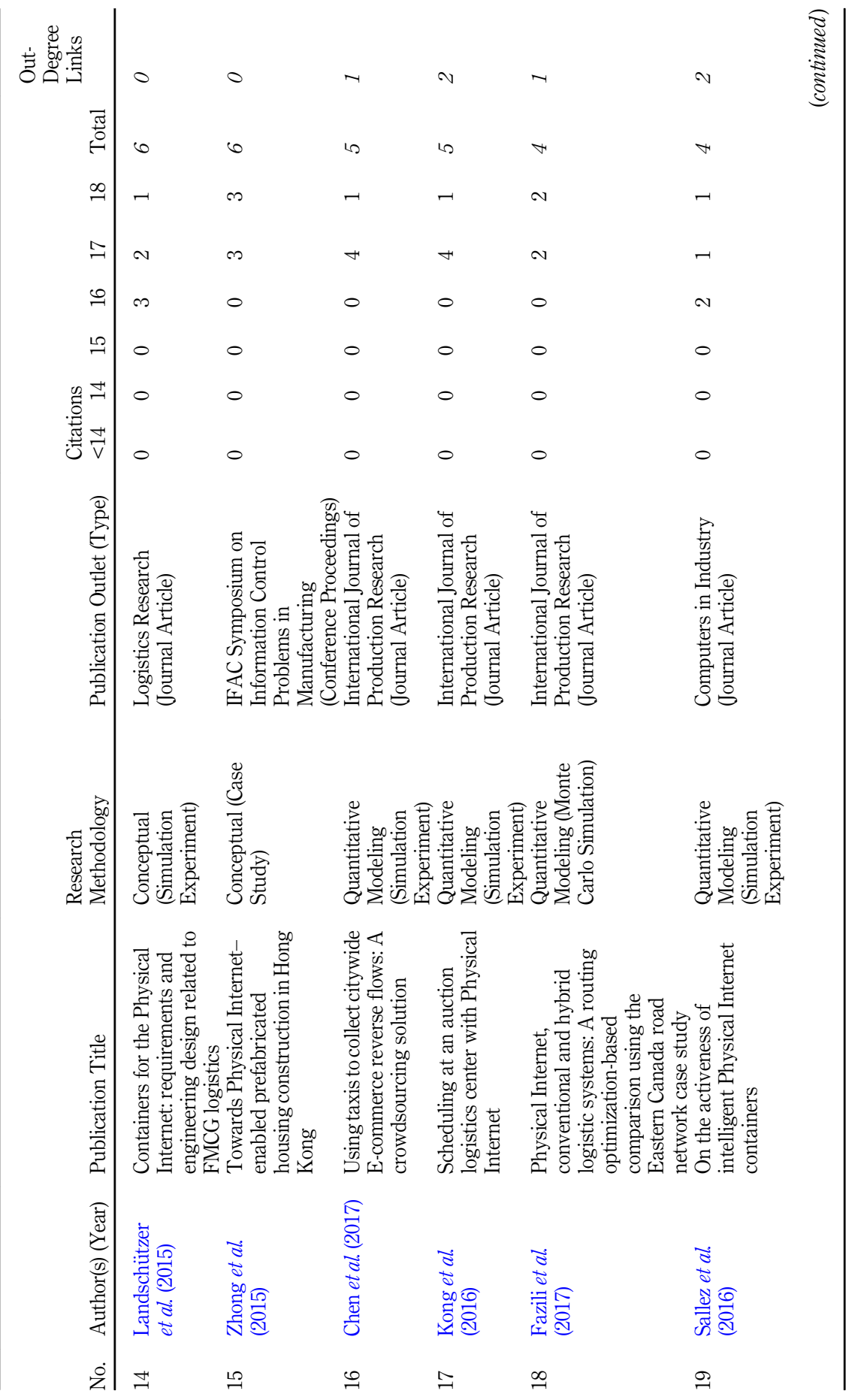




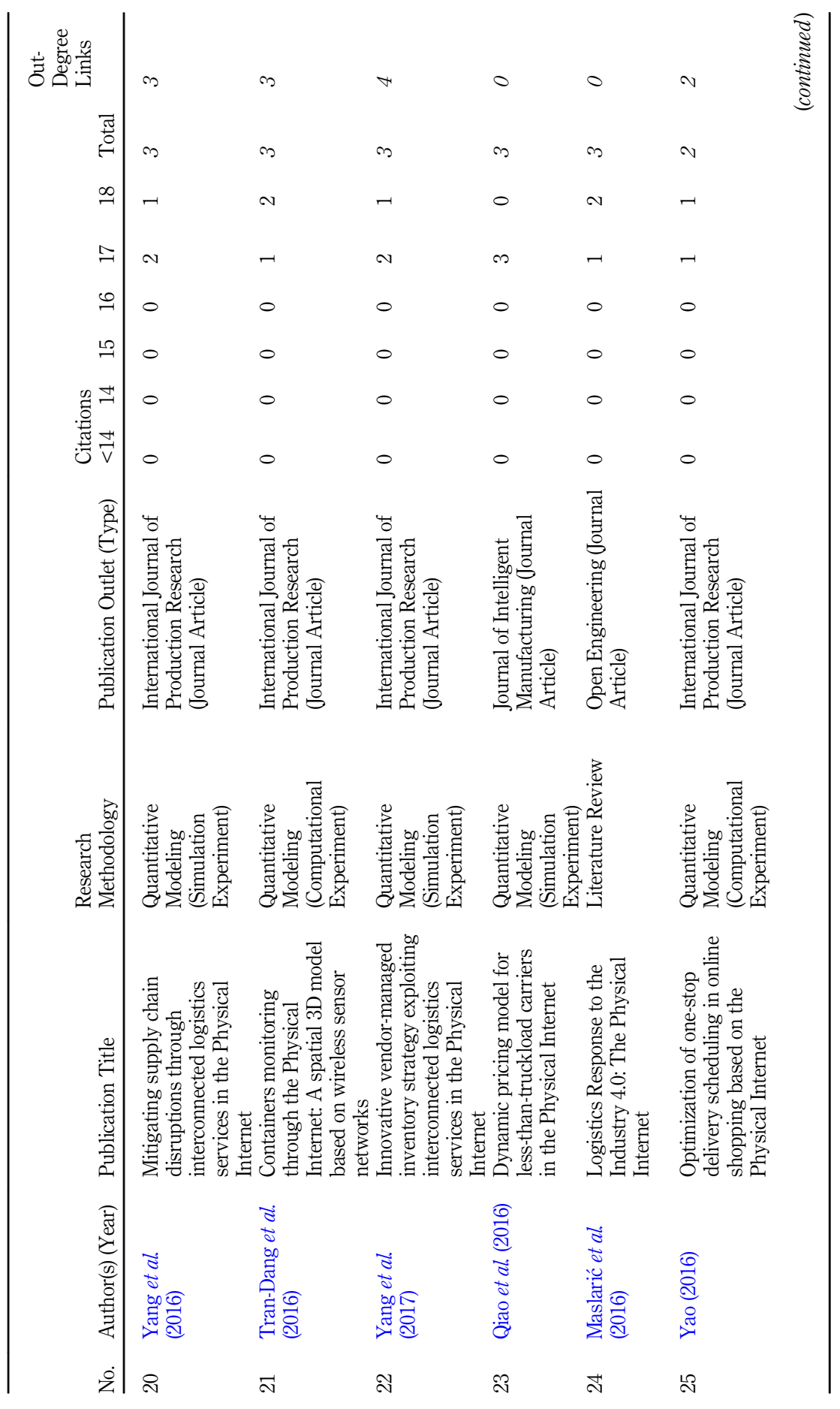

PI as a new supply chain paradigm

285

Table AI. 


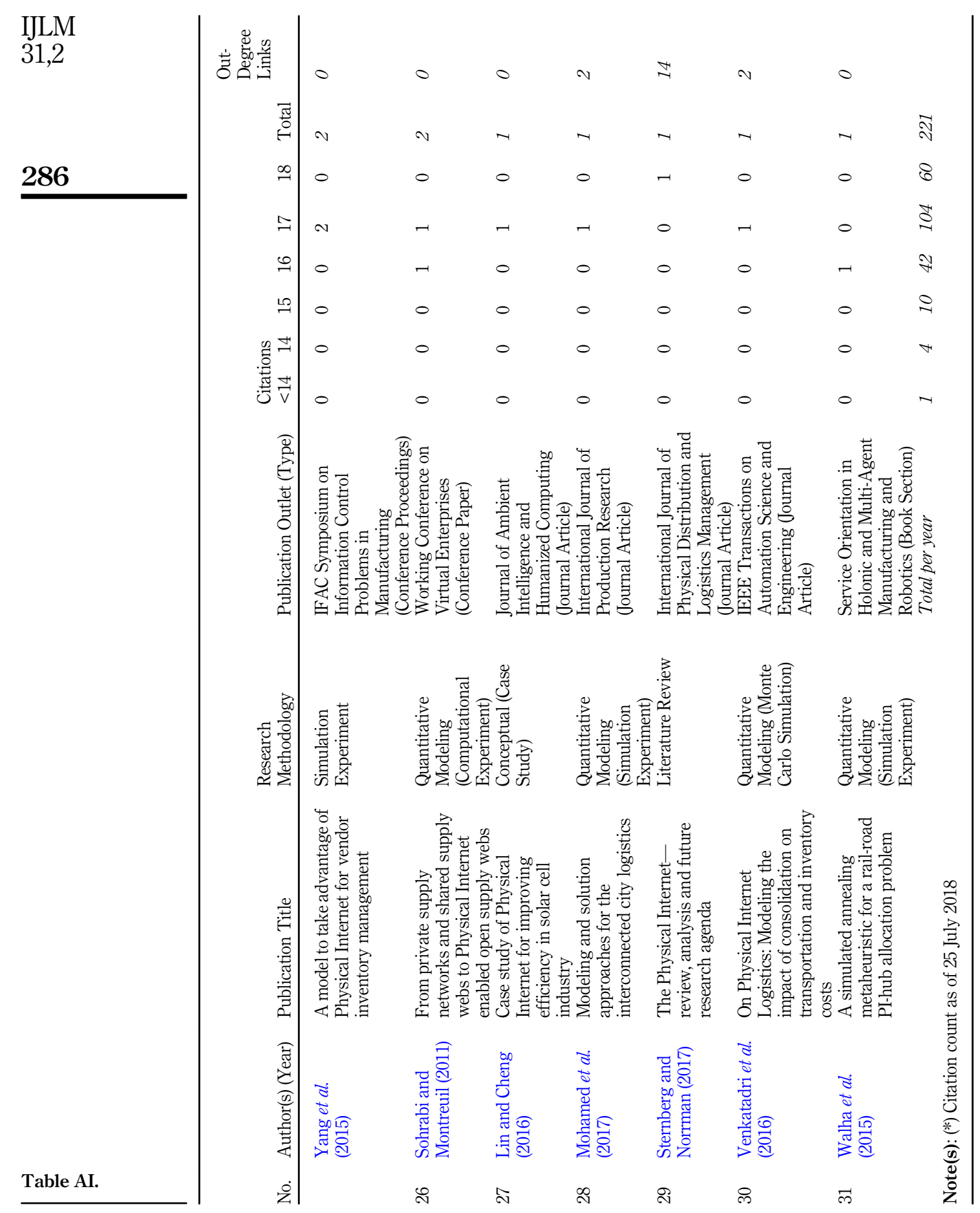


Required

Characteristics (Crucial

for PI)

Business models Innovative business models built on the principles of the PI

Cooperation models Cooperation models with fair sharing of revenues

Legal framework

Seamless, secure and confidential data exchange

Transit centers, hubs

Vehicle usage

optimization

Modular containers (transport containers, handling containers, packaging containers)
Legal security, seamless international transport Open, shared and secure protocols for data exchange, mechanisms to restrict access to data on goods and information about delivery status Creating modern and open transit centers, fully functional design, efficient and effective cargo handling Shared, fully loaded, energy-efficient vehicles using relays Creating shared, modular, sustainable, robust, lightweight scalable containers
Complicated, national regulations

Proprietary solutions, security breaches, measures which alleviate the theft of data and goods

Using and building nonstandardized proprietary transit centers, hubs

Empty or partly loaded vehicles, unstandardized, one-way

Using proprietary, unsustainable solutions
Publications

Montreuil (2012); Oktaei

et al. (2014, 2015), Rougès and Montreuil (2014)

Koulougli et al. (2015);

Franklin and Spinler

(2011); Sohrabi et al.,(2015); Xu et al. (2013); Verstrepen and Van Den Bossche (2013); Genta and Cruijssen (2013); Rossi (2012); Cruijssen (2012); Jacobs et al. (2014); Eye For Transport (2011) Biermasz and Louws (2014)

Tretola and Verdino (2014, 2015); Hakimi et al. (2012); Hofman (2015)

Montreuil (2010, 2012); Ballot et al. (2012); Meller et al. (2012b); Pach et al. (2014); Walha et al. (2015)

Lin et al. (2014); Xu et al. (2013); Furtado and Frayret (2014) Lin et al. (2014); Landschützer et al. (2015); Meller et al. (2012a); TranDang et al. (2015); Sarraj et al. (2014a); Sallez et al. (2015b); Sallez et al. (2016); Zhang et al. (2016)
PI as a new supply chain paradigm
Table AII.

PI themes emerging from the literature review

\section{Appendix 2}

The supplementary material for Appendix 2 can be found here: https://www.researchgate.net/ publication/347945858_The_Physical_Internet_as_a_New_Supply_Chain_Paradigm_A_Systematic_ Literature_Review_and_a_Comprehensive_Framework_Appendix_B.

\section{Corresponding author}

Horst Treiblmaier can be contacted at: horst.treiblmaier@modul.ac.at

For instructions on how to order reprints of this article, please visit our website:

www.emeraldgrouppublishing.com/licensing/reprints.htm

Or contact us for further details: permissions@emeraldinsight.com 